OPEN ACCESS

Edited by:

Matiram Pun,

University of Calgary, Canada

Reviewed by:

Fernando A. Moraga

Catholic University of the North, Chile

Sanjeeb Bhandari,

Virginia Tech Carilion Schoo

of Medicine and Research Institute,

United States

*Correspondence:

Jui-Lin Fan

mickey.fan@auckland.ac.nz

Specialty section:

This article was submitted to

Environmental, Aviation and Space

Physiology,

a section of the journal

Frontiers in Physiology

Received: 15 October 2020

Accepted: 18 January 2021

Published: 24 February 2021

Citation:

Fan J-L, Wu TY, Lovering AT,

Nan L, Bang WL and Kayser B (2021) Differential Brain and Muscle Tissue Oxygenation Responses to Exercise

in Tibetans Compared to Han

Chinese. Front. Physiol. 12:617954. doi: 10.3389/fphys.2021.617954

\section{Differential Brain and Muscle Tissue Oxygenation Responses to Exercise in Tibetans Compared to Han Chinese}

\author{
Jui-Lin Fan ${ }^{*}$, Tian Yi Wu ${ }^{2,3}$, Andrew T. Lovering ${ }^{4}$, Liya Nan ${ }^{3}$, Wang Liang Bang ${ }^{3}$ and \\ Bengt Kayser ${ }^{5}$ \\ 1 Department of Physiology, Faculty of Medical and Health Sciences, University of Auckland, Auckland, New Zealand, \\ ${ }^{2}$ Research Center for High Altitude Medicine, Tibet University Medical College, Lhasa, China, ${ }^{3}$ National Key Laboratory \\ of High Altitude Medicine, Xining, China, ${ }^{4}$ Department of Human Physiology, University of Oregon, Eugene, OR, \\ United States, ${ }^{5}$ Institute of Sport Sciences, University of Lausanne, Lausanne, Switzerland
}

The Tibetans' better aerobic exercise capacity at altitude remains ill-understood. We tested the hypothesis that Tibetans display better muscle and brain tissue oxygenation during exercise in hypoxia. Using near-infrared spectrometry (NIRS) to provide indices of tissue oxygenation, we measured oxy- and deoxy-hemoglobin $\left(\left[\mathrm{O}_{2} \mathrm{Hb}\right]\right.$ and $[\mathrm{HHb}]$, respectively) responses of the vastus lateralis muscle and the right prefrontal cortex in ten Han Chinese and ten Tibetans during incremental cycling to exhaustion in a pressure-regulated chamber at simulated sea-level (air at 1 atm: normobaric normoxia) and 5,000 m (air at 0.5 atm: hypobaric hypoxia). Hypoxia reduced aerobic capacity by $\sim 22 \%$ in both groups ( $d=0.8, p<0.001$ vs. normoxia), while Tibetans consistently outperformed their Han Chinese counterpart by 32\% in normoxia and hypoxia ( $d=1.0$, $p=0.008)$. We found cerebral $\left[\mathrm{O}_{2} \mathrm{Hb}\right]$ was higher in Tibetans at normoxic maximal effort compared Han $(p=0.001)$, while muscle $\left[\mathrm{O}_{2} \mathrm{Hb}\right.$ ] was not different $(p=0.240)$. Hypoxic exercise lowered muscle $\left[\mathrm{O}_{2} \mathrm{Hb}\right]$ in Tibetans by a greater extent than in Han (interaction effect: $p<0.001$ vs. normoxic exercise). Muscle $\left[\mathrm{O}_{2} \mathrm{Hb}\right]$ was lower in Tibetans when compared to Han during hypoxic exercise $(d=0.9, p=0.003)$, but not during normoxic exercise $(d=0.4, p=0.240)$. Muscle $[\mathrm{HHb}]$ was not different between the two groups during normoxic and hypoxic exercise $(p=0.778)$. Compared to Han, our findings revealed a higher brain tissue oxygenation in Tibetans during maximal exercise in normoxia, but lower muscle tissue oxygenation during exercise in hypoxia. This would suggest that the Tibetans privileged oxygenation of the brain at the expense of that of the muscle.

Keywords: Tibetans, exercise performance, hypoxia, muscle tissue oxygenation, cerebral tissue oxygenation

\section{INTRODUCTION}

Aerobic performance is an important determinant of one's ability to thrive at high altitude, yet the exact mechanisms limiting aerobic performance at high altitude remain poorly understood. Tibetans are renowned for their superior exercise performance at high altitude compared to their Western and Han Chinese counterparts (Wu et al., 2005; Marconi et al., 2006; Wu and Kayser, 2006; 
Gilbert-Kawai et al., 2014; Kayser et al., 2019). While this remarkable performance has been largely attributed to better systemic oxygen $\left(\mathrm{O}_{2}\right)$ transport in Tibetans, it is unknown whether this translates into better muscle and/or brain tissue oxygenation during exercise.

Within the skeletal muscles, Tibetans and Sherpas display lower mitochondrial volume density and muscle fiber crosssectional area, and higher muscle capillary density and myoglobin concentration compared to lowlanders (Kayser et al., 1991, 1996; Erzurum et al., 2007). Furthermore, Tibetans have been reported to display lower $\mathrm{O}_{2}$ consumption for a given exercise workload compared to lowlanders, suggesting a better $\mathrm{O}_{2}$ economy ( $\mathrm{Ge}$ et al., 1994). But how these differences in muscle ultrastructure and $\mathrm{O}_{2}$ economy relate to oxidative metabolism in the working muscle has not been investigated. By comparing muscle tissue oxygenation in Tibetans and their Han Chinese counterparts during exercise in normoxia and hypoxia, we aimed to gain further insight into the functional differences in skeletal muscle oxidative metabolism between these two groups.

Near-infrared spectroscopy (NIRS) provides a non-invasive method of assessing changes in muscle tissue oxygenation during exercise (Perrey and Ferrari, 2017). By determining the relative changes in oxy- and deoxy-hemoglobin concentrations $\left(\left[\mathrm{O}_{2} \mathrm{Hb}\right]\right.$ and $[\mathrm{HHb}]$, respectively), muscle NIRS signals provide unique insights into $\mathrm{O}_{2}$ balance (and therefore oxidative metabolism) in the working muscle (Grassi and Quaresima, 2016; Perrey and Ferrari, 2017). In the brain, NIRS-determined capillary oxygenation is functionally related to the balance between $\mathrm{O}_{2}$ saturation of arterial and venous blood, and has been shown to reflect cerebral capillary oxygenationlevel-dependent changes (Rasmussen et al., 2007). Studies have reported an association between performance and NIRSderived cerebral tissue deoxygenation in severe hypoxia (arterial oxygen saturation $\left.\left[\mathrm{SaO}_{2}\right]<75 \%\right)$ during repeated sprints (Smith and Billaut, 2010), incremental exercise (Subudhi et al., 2007; Peltonen et al., 2009), and static maximal or sub-maximal muscle contraction to exhaustion (Rasmussen et al., 2007; Rupp and Perrey, 2009; Vogiatzis et al., 2011; Millet et al., 2012).

It has been speculated that cerebral tissue oxygenation may play a pivotal role in the limitation of performance during exercise in severe hypoxia [(Kjaer et al., 1999; Amann et al., 2006, 2007; Rasmussen et al., 2010), see Fan and Kayser (2016) for review]. During incremental cycling at 3,658 $\mathrm{m}$, Tibetans have been reported to exhibit higher internal carotid blood flow and cerebral $\mathrm{O}_{2}$ delivery compared to Han Chinese (Huang et al., 1992). Whether these differences translate to higher cerebral tissue oxygenation, and thereby could partly account for the superior performance in Tibetans is unknown.

The purpose of this study was to compare the muscle and cerebral tissue oxygenation responses to exercise in a pressureregulated chamber in normobaric normoxia (air at 1 atm, to simulate sea-level) and hypobaric hypoxia (air at $0.5 \mathrm{~atm}$, equivalent to $\sim 5,000 \mathrm{~m}$ altitude) in Tibetans and Han Chinese living in Xining $(2,260 \mathrm{~m})$ of the Qinghai Province, People's Republic of China. We tested the hypothesis that when compared to Han Chinese, Tibetans would exhibit higher brain and muscle tissue oxygenation during exercise in hypoxia, but not during exercise in normoxia.

\section{MATERIALS AND METHODS}

\section{Participants}

Forty male participants were initially recruited into this study. Following saline-contrast echocardiography screening to exclude participants with a patent foramen ovale (see below), twenty male participants completed this study, consisting of ten Han Chinese and ten Tibetans (Table 1). The Tibetan group consisted of individuals of Tibetan descent who were born and raised at altitudes of $>3,500 \mathrm{~m}$ and currently residing in Xining of the Qinghai Province, Peoples Republic of China, while the Han Chinese group were all second-generation permanent residents of Xining of the Qinghai Province, Peoples Republic of China. To minimize the confounding influence of training status and physical activity, we recruited recreationally active individuals.

All the participants were judged healthy by medical history, physical examination, resting electrocardiogram, echocardiogram and respiratory function tests and were not taking any medication. The participants were informed of the experimental procedures and potential risks involved in the study before their written consent was obtained. The study was approved by the University of Oregon Institutional Review Board and the Qinghai High Altitude Medical Science Institutional Committee on Human Research and complied with the Declaration of Helsinki.

\section{Saline-Contrast Echocardiography Screening}

All the participants underwent saline-contrast echocardiography with and without performing a Valsalva maneuver to screen for the presence of patent foramen ovale (PFO). The foramen ovale is an interatrial communication which allows blood flow to bypass the pulmonary circulation during fetal life but normally closes after birth. Importantly, the presence of a PFO in adults has been shown to elevate resting alveolar-arterial $\mathrm{O}_{2}$ gradient (i.e., reduced pulmonary gas exchange efficiency) and lower $\mathrm{SaO}_{2}$ during maximal exercise in normoxia (Lovering et al., 2011). Likewise, the presence of a PFO is believed to exacerbate arterial

TABLE 1 | Participant characteristics of Han Chinese and Tibetans (values concern acute normobaric normoxic condition).

\begin{tabular}{|c|c|c|c|}
\hline & Han Chinese & Tibetan & $p$-value \\
\hline$n$ & 10 & 10 & \\
\hline Age(years) & $23.3 \pm 1.6$ & $21.2 \pm 3.1$ & 0.078 \\
\hline Height $(\mathrm{cm})$ & $173.9 \pm 6.4$ & $171.0 \pm 6.1$ & 0.531 \\
\hline Body mass (kg) & $62.2 \pm 7.7$ & $64.4 \pm 7.7$ & 0.314 \\
\hline $\mathrm{BMI}\left(\mathrm{kg} \mathrm{m}^{-2}\right)$ & $20.5 \pm 1.9$ & $22.0 \pm 1.9$ & 0.108 \\
\hline$\dot{\mathrm{V}}_{2}$ peak $\left(\mathrm{ml} \cdot \mathrm{min}^{-1} \cdot \mathrm{kg}\right)$ & $35.9 \pm 4.1$ & $38.9 \pm 10.2$ & 0.408 \\
\hline$[\mathrm{Hb}]\left(\mathrm{g} \cdot \mathrm{L}^{-1}\right)$ & $16.5 \pm 1.7$ & $16.0 \pm 3.2$ & 0.724 \\
\hline Hct $(\%)$ & $55.7 \pm 2.1$ & $56.2 \pm 3.5$ & 0.658 \\
\hline
\end{tabular}

BMI, body mass index; $\mathrm{VO}_{2}$ peak, $\mathrm{O}_{2}$ uptake at 1 atm; [Hb], Hemoglobin concentration; Hct, hematocrit concentration. 
hypoxemia in severe chronic obstructive pulmonary disease patients at rest (Allemann et al., 2006; Brenner et al., 2015; Elliott et al., 2015). Therefore, to avoid the possible confounding influence of a PFO, those who were determined to have a PFO were excluded from the study. Similarly as previously found in Sherpas living at high altitude (Foster et al., 2014), the prevalence of PFO was $50 \%$ in our cohort of Tibetans.

\section{Experimental Design}

The participants visited the laboratory on three occasions. After a full familiarization with the experimental procedures outlined below (visit one), the participants underwent two experimental exercise sessions (visits two and three) in a pressure-regulated chamber $(3 \mathrm{~m} \times 8 \mathrm{~m} \times 3 \mathrm{~m}$ hyper- and hypobaric pressure chamber, Shanghai Far East Petroleum Machinery Co., China): (i) normobaric normoxia (simulated sea-level by increasing the chamber pressure to $1 \mathrm{~atm}$ ), and (ii) hypobaric hypoxia (simulated $5,000 \mathrm{~m}$ by decreasing the chamber pressure to $0.5 \mathrm{~atm}$ ), in a randomized, single-blinded, balanced fashion. Due to technical limitations with the chamber, there was a mild increase in fraction of inspired $\mathrm{O}_{2}\left(\mathrm{FIO}_{2}\right)$ from 0.209 to $0.22-0.24$ resulting in a mild increase in inspired $\mathrm{O}_{2}$ (see methodological considerations).

For the normobaric normoxia and hypobaric hypoxia sessions, the experimental protocol was comprised of: (i) $20 \mathrm{~min}$ instrumentation; (ii) 4 min hyperoxic exposure, breathing $100 \%$ $\mathrm{O}_{2}$ through a face mask, followed by a 3-min washout period to ensure all the variables returned to pre- $\mathrm{O}_{2}$ breathing values; (iii) 4 min resting baseline; (iv) step-incremental cycling until exhaustion; (v) $5 \mathrm{~min}$ recovery; and (vi) ramp-incremental cycling until exhaustion. All of the experimental sessions were conducted at the National Key Laboratory of High Altitude Medicine in the city of Xining of the Qinghai Province, People's Republic of China. Before each experimental session, all participants were asked to abstain from caffeine for $12 \mathrm{~h}$, and alcohol for $24 \mathrm{~h}$.

\section{Exercise Tests}

\section{Step-Incremental Cycling Until Exhaustion}

Seated on a reclining ergometer tilted into a left lateral position (Ergoselect 1000, Ergoline GmbH, Bitz, Germany), the participants were instructed to begin cycling at 70 watts, at a pedaling rate of $70 \mathrm{rpm}$. The work rate was increased by 30 watts every $3 \mathrm{~min}$ thereafter until the participant reached voluntary exhaustion.

\section{Ramp Incremental Cycling to Exhaustion}

Following a 5-min recovery period whilst positioned on the ergometer, the participants were instructed to begin cycling at 0 watts, at a pedaling rate of $70 \mathrm{rpm}$. The work rate was increased by 0.5 watts every second (i.e., equivalent of 30 watts. $\mathrm{min}^{-1}$ ) thereafter until the participant reached voluntary exhaustion.

\section{Measurements \\ Respiratory Variables}

Throughout the experimental protocol, the participants wore a facemask attached to a spirometer (TripleV-volume sensor,
CareFusion, San Diego, CA, United States), from which expired gases and breath-by-breath respiratory flow was monitored using a metabolic cart (Jaeger, CareFusion, San Diego, CA, United States). Ventilation ( $\dot{\mathrm{V} E}), \mathrm{O}_{2}$ uptake $\left(\dot{\mathrm{V}}_{2}\right)$, expired $\left.\mathrm{CO}_{2}(\dot{\mathrm{V} C O})_{2}\right)$, and respiratory exchange ratio (RER) were then calculated by the metabolic cart and expressed in either L. $\mathrm{min}^{-1}$ BTPS (for VE) or mL.min ${ }^{-1}$ STPD $\left(\dot{\mathrm{VO}} 2\right.$ and $\left.\dot{\mathrm{V}} \mathrm{CO}_{2}\right)$.

\section{Cardiovascular and Cerebrovascular Variables}

Continuous beat-to-beat blood pressure was monitored using finger plethysmography (Finometer MIDI, Finapres Medical Systems, Amsterdam, Netherlands), from which mean blood pressure (BP) was derived from the timed-average of the $\mathrm{BP}$ waveform. Peripheral $\mathrm{O}_{2}$ saturation $\left(\mathrm{SpO}_{2}\right)$ was measured from the right side of the forehead using pulse oximetry (N-200, Nellcor Inc., Hayward, CA, United States). A threelead electrocardiogram was used to determine heart rate (HR). Stroke volume (SV) was estimated using transthoracic electrical bioimpedance cardiography (PhysioFlow ${ }^{\circledR}$, Manatec PF07 Enduro, Paris, France). Cardiac output (CO) was subsequently calculated by multiplying SV with HR.

Bilateral middle cerebral artery blood velocities (MCAv, an index of cerebral blood flow) were measured in the middle cerebral artery using a 2-MHz pulsed Doppler ultrasound system (ST3, Spencer Technology, Seattle, OR, United States). The Doppler ultrasound probes were positioned over the temporal windows and held in place with an adjustable plastic headband. The MCAv signals were acquired at depths ranging from 43 to $54 \mathrm{~mm}$. Signal quality was optimized, and an M-mode screen shot was recorded to facilitate subsequent probe placements. In our hands, day-to-day reproducibility of MCAv has a coefficient of variation of $<10 \%$. The bilateral MCAv were averaged using the following equation to represent global cerebral blood flow (CBF) during rest and exercise:

$$
\text { mean MCAv }=\left[\frac{\text { left } \mathrm{MCAv}+\text { right MCAv }}{2}\right]
$$

\section{Muscle and Cerebral Tissue Oxygenation}

Muscle tissue oxygenation in the left vastus lateralis muscle $(\sim 15 \mathrm{~cm}$ proximal and $5 \mathrm{~cm}$ lateral to the superior border of the patella) was measured by monitoring changes in $\left[\mathrm{O}_{2} \mathrm{Hb}\right]$ and $[\mathrm{HHb}]$ concentrations obtained using spatially resolved, continuous wave near-infrared spectroscopy (NIRS, Oxymon MKIII, Artinis, Zetten, Netherlands). For the muscle tissue oxygenation, a source-detector spacing of $3.8 \mathrm{~cm}$ and a differential pathlength factor of 4.0 were used (Duncan et al., 1995). Cerebral tissue oxygenation in the left prefrontal lobe was assessed with an additional NIRS channel of the same instrument. Both muscle and cerebral NIRS channels were zeroed at rest and expressed as absolute values. For the cerebral tissue oxygenation, source-detector spacing was set at $4.1 \mathrm{~cm}$ and data obtained from the optodes were used to calculate changes in $\left[\mathrm{O}_{2} \mathrm{Hb}\right]$ and $[\mathrm{HHb}]$ with a differential pathlength factor (DPF) calculated using the formula: $\mathrm{DPF}=4.99+0.067 \times$ age $^{0.814}$ (Duncan et al., 1995). Total $\mathrm{Hb}$ ([totHb]) was calculated using the equation:

$$
[\text { tot } \mathrm{Hb}]=\left[\mathrm{O}_{2} \mathrm{Hb}\right]+[\mathrm{HHb}]
$$


The cerebral and muscle $\left[\mathrm{O}_{2} \mathrm{Hb}\right],[\mathrm{HHb}]$, and [totHb] are expressed as absolute values ( $\mu \mathrm{Mol})$.

\section{Arterial Blood Gases}

Arterial blood gas samples were obtained from a 22-gauge arterial catheter placed into a radial artery; blood samples $(2 \mathrm{~mL})$ were taken over approximately five cardiac cycle periods into a pre-heparinized syringe. Following standardized calibration, all blood samples were analyzed using an arterial blood-gas analyzing system (ABL $77 \mathrm{Sci}$, Radiometer, Copenhagen, Denmark) for $\mathrm{pH}$, partial pressure of arterial $\mathrm{O}_{2}\left(\mathrm{PaO}_{2}\right)$ and $\mathrm{CO}_{2}\left(\mathrm{PaCO}_{2}\right)$, arterial $\mathrm{O}_{2}$ saturation $\left(\mathrm{SaO}_{2}\right)$, hemoglobin concentration ([Hb]) and hematocrit (Hct). Arterial $\mathrm{O}_{2}$ content $\left(\mathrm{CaO}_{2}\right)$ was subsequently calculated using the equation:

$$
\mathrm{CaO}_{2}=[\mathrm{Hb}] \times 1.36 \times\left[\frac{\mathrm{SaO}_{2}}{100}\right]+\mathrm{PaO}_{2} \times 0.003
$$

Aural temperature from the right ear was recorded as a surrogate of core body temperature. The blood gas values were temperature corrected (Kelman and Nunn, 1966; Severinghaus, 1966).

\section{Energy Economy}

Energy expended (EE) was calculated using the equation (Moseley and Jeukendrup, 2001):

$\mathrm{EE}=\left[\left(\dot{\mathrm{V}} \mathrm{O}_{2} \times 3.869\right)+\left(\dot{\mathrm{V} C O}{ }_{2} \times 1.195\right) \times(4.186 / 60) \times 1000\right]$

where, EE is energy expended in $\mathrm{J} . \mathrm{s}^{-1}, \mathrm{~V}_{2}$ is $\mathrm{O}_{2}$ uptake in L.min ${ }^{-1}$ and $\dot{\mathrm{V} C O}{ }_{2}$ is the $\mathrm{CO}_{2}$ expired in L.min ${ }^{-1}$.

Gross efficiency (GE) during steady-state exercise was calculated using the equation (Moseley and Jeukendrup, 2001):

$$
\mathrm{GE}=\text { Work rate } / \mathrm{EE} \times 100
$$

Where GE is gross efficiency in percentage, work rate is in watts, and EE is energy expended in J.s ${ }^{-1}$.

Exercise economy (EC) was calculated as power output divided by $\mathrm{O}_{2}$ uptake and expressed as kJ.L $\mathrm{L}^{-1}$.

Except for $\dot{\mathrm{VE}}, \dot{\mathrm{V}}_{2}, \dot{\mathrm{V} C O}{ }_{2}$ and RER which were recorded on the metabolic cart, all analog data were sampled and recorded at $200 \mathrm{~Hz}$ on a computer for off-line analysis (Powerlab 16/30, ADInstruments, Dunedin, New Zealand).

\section{Statistical Analysis}

Unpaired $t$ test with Welch's correction was used to compare the participants' characteristics between Han Chinese and Tibetan (Prism 8, GraphPad Software, San Diego, CA, United States). Analyses of resting parameters were performed on averaged data from the last $2 \mathrm{~min}$ of the baseline period, and from the last $1 \mathrm{~min}$ of the hyperoxic exposure and recovery period. During exercise, the mean values during the last $30 \mathrm{~s}$ of workload (i.e., 70 watts, 100 watts, 130 watts, etc.), and the mean value of the last $30 \mathrm{~s}$ of the ramp incremental exercise for maximal exercise effort (MAX) were extracted for analysis.

The main effects of experimental condition (normoxia vs. hypoxia) and group (Han Chinese vs. Tibetans) on arterial blood gases, cardiorespiratory variables, cerebral haemodynamics and muscle tissue oxygenation during $100 \% \mathrm{O}_{2}$ breathing, at rest, during steady-state exercise (70 watts, 100 watts, 130 watts, and 160 watts), recovery and maximal exercise effort were assessed using mixed linear model analysis (SPSS Statistics version 23, IBM Corporation, Armonk, NY, United States). For significant effects and interaction between hypoxia effect and group effect, post hoc tests were performed using Sidak's adjustment for multiple comparisons ( $\alpha$-level of 0.05$)$. In addition to $p$-values, Cohen's $d$ values (effect size) are reported for altitude and group effects. Cohen's $d$ value was calculated using the formula (Cohen, 1977):

$$
d=\left[\frac{\mathrm{M}_{1}-\mathrm{M}_{2}}{\sigma_{\text {pooled }}}\right]
$$

where, $M_{1}$ and $M_{2}$ are means of group 1 and 2; $\sigma_{\text {pooled }}$ is the standard deviation of the pooled data. The effect sizes were classified as (Sullivan and Feinn, 2012): negligible $(d<0.2)$; small $(d \geq 0.2)$; medium $(d \geq 0.5)$; large $(d \geq 0.8)$; and very large $(d \geq 1.3)$. Data are reported as mean \pm SD in text, tables and figures.

\section{RESULTS}

\section{Hyperoxia}

In hypoxic conditions, $100 \% \mathrm{O}_{2}$ breathing increased muscle $\left[\mathrm{O}_{2} \mathrm{Hb}\right]$ by $\sim 2.9 \mu \mathrm{M}$ in both groups $(F=7.8, d=0.8, p=0.012)$, while neither muscle $[\mathrm{HHb}](F=1.3, p=0.264)$ nor [totHb] were affected $(F=1.2, p=0.275)$. There was a tendency for muscle $[\mathrm{HHb}$ ] to be lower by $\sim 2.1 \mu \mathrm{M}$ in Tibetans during $100 \%$ $\mathrm{O}_{2}$ breathing $(F=3.5, d=0.4, p=0.071$ vs. Han Chinese, Figure 4). In both groups, $100 \% \mathrm{O}_{2}$ breathing decreased MCAv by $\sim 7.3 \mathrm{~cm} . \mathrm{s}^{-1}$ in hypoxia compared to normoxia $(F=5.4$, $d=0.5, p=0.032$ ), while no significant group effects were observed $(p>0.05)$.

\section{Performance}

Hypoxia reduced aerobic capacity by $\sim 22 \%$ in both Han Chinese and Tibetans compared to their normoxic values $(F=31.5$, $d=0.8, p<0.001$, Figure 1). Irrespective of the experimental condition, Tibetans performed significantly better than their Han Chinese counterparts by $\sim 32 \%$ during incremental exercise to exhaustion $(F=8.8, d=1.0, p=0.008)$.

\section{Resting, Steady-State Exercise and Recovery \\ Arterial Blood Gases}

At rest, lowering barometric pressure reduced $\mathrm{PaO}_{2}$ by $\sim 85.6 \mathrm{mmHg}(F=1778.1), \mathrm{PaCO}_{2}$ by $\sim 6.3 \mathrm{mmHg}(F=112.7)$, $\mathrm{SaO}_{2}$ by $\sim 16.8 \%(F=132.8)$ and $\mathrm{CaO}_{2}$ by $\sim 3.0 \mathrm{~mL} \mathrm{O}_{2} . \mathrm{dl}^{-1}$ $(F=10.51)$, and elevated resting $\mathrm{pH}$ by $\sim 0.05(F=60.5)$ in both 


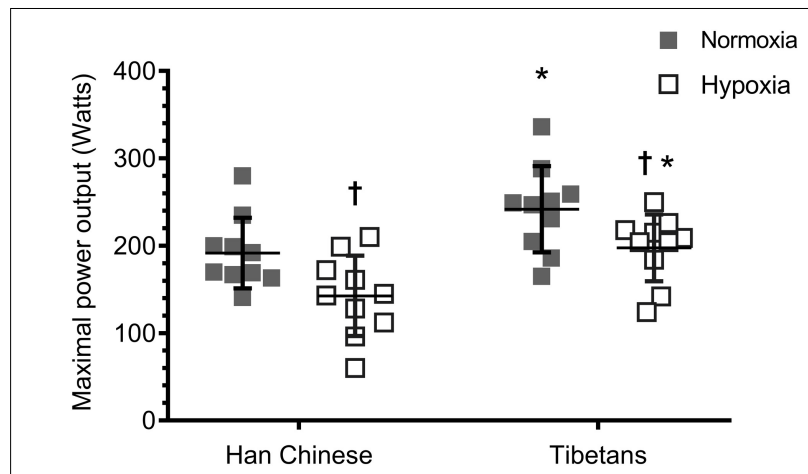

FIGURE 1 | Aerobic capacity during ramp incremental cycling to exhaustion in Han Chinese and Tibetans. *different from Han Chinese, $p<0.05$; ${ }^{\dagger}$ different from normoxia. Data expressed mean \pm SD.

Han Chinese and Tibetans $(d>1.0$ and $p<0.01$ vs. normoxia for all, Table 2).

On average, hypoxia reduced $\mathrm{PaO}_{2}$ by $\sim 76.3 \mathrm{mmHg}$ throughout step-incremental cycling $(F=8682.7, d=2.0)$,
$\mathrm{PaCO}_{2}$ by $\sim 13.7 \mathrm{mmHg}(F=554.4, d=1.5), \mathrm{SaO}_{2}$ by $\sim 17.5 \%$ $(F=2240.9, d=1.0), \mathrm{CaO}_{2}$ by $\sim 3.2 \mathrm{~mL} \mathrm{O} \cdot \mathrm{dl}^{-1}(F=81.8$, $d=1.1)$ in both Han Chinese and Tibetans, while it elevated [Hb] by $\sim 0.8$ g.L ${ }^{-1}(F=9.1, d=0.5)$ and $\mathrm{pH}$ by $\sim 0.09(F=92.9$, $d=1.5, p<0.001$ for all, Table 3). There was no other difference between Han Chinese and Tibetans in any of the arterial blood gas parameters during exercise in both hypoxic and normoxic conditions ( $p>0.05$, Table 3$)$.

During maximal effort, hypoxia lowered $\mathrm{PaO}_{2}$ by $\sim 72.8 \mathrm{mmHg}(F=1569.0, d=1.9), \mathrm{PaCO}_{2}$ by $\sim 16.7 \mathrm{mmHg}$ $(F=259.2, d=1.7), \mathrm{SaO}_{2}$ by $\sim 16.6 \%(F=66.1, d=1.9), \mathrm{CaO}_{2}$ by $\sim 2.9 \mathrm{~mL} \mathrm{O}_{2} \cdot \mathrm{dl}^{-1}$ in both groups $(F=12.3, d=1.0)$, while $\mathrm{pH}$ was elevated by $\sim 0.10$ compared to normoxia $(F=66.1, d=1.7$, $p<0.001$ for all, Table 3$)$. There were no group effects on arterial blood gas parameters ( $p>0.05$, Table 3$)$.

\section{Cardiorespiratory}

In both Han Chinese and Tibetans, exposure to hypoxia elevated resting HR by $\sim 19.6 \mathrm{~b} \cdot \mathrm{min}^{-1}(F=58.5, d=1.2)$, CO by $\sim 1.0 \mathrm{~L} \cdot \mathrm{min}^{-1}(F=6.1, d=0.6)$ and lowered $\mathrm{SpO}_{2}$ by $\sim 17.7 \%$ $(F=152.8, d=1.8)$ compared to normoxia ( $p<0.05$ vs.

TABLE 2 | Resting cardiorespiratory, cerebral haemodynamics and muscle tissue oxygenation in Han Chinese and Tibetans in normobaric normoxia and hypobaric hypoxia.

\begin{tabular}{|c|c|c|c|c|c|c|c|}
\hline \multirow{3}{*}{$\begin{array}{l}n \\
\text { Arterial blood gases }\end{array}$} & \multicolumn{2}{|c|}{ Han Chinese } & \multicolumn{2}{|c|}{ Tibetan } & \multicolumn{3}{|c|}{ Main effects ( $p$-values) } \\
\hline & \multicolumn{2}{|c|}{10} & \multicolumn{2}{|c|}{10} & \multirow[b]{2}{*}{ Condition } & \multirow[b]{2}{*}{ Group } & \multirow[b]{2}{*}{ Interaction } \\
\hline & Normoxia & Hypoxia & Normoxia & Hypoxia & & & \\
\hline $\mathrm{PaO}_{2}(\mathrm{mmHg})$ & $128.8 \pm 7.5$ & $45.6 \pm 4.9+$ & $135.1 \pm 8.9$ & $47.2 \pm 7.3+$ & 0.000 & 0.158 & 0.263 \\
\hline $\mathrm{PaCO}_{2}(\mathrm{mmHg})$ & $40.2 \pm 5.2$ & $33.0 \pm 3.8+$ & $37.7 \pm 4.2$ & $32.4 \pm 4.1 \dagger$ & 0.000 & 0.417 & 0.126 \\
\hline $\mathrm{pH}$ & $7.35 \pm 0.02$ & $7.41 \pm 0.03+$ & $7.37 \pm 0.03$ & $7.41 \pm 0.03+$ & 0.000 & 0.319 & 0.091 \\
\hline $\mathrm{SaO}_{2}(\%)$ & $98.8 \pm 0.2$ & $81.2 \pm 4.0 \dagger$ & $99.0 \pm 0.1$ & $83.0 \pm 8.4+$ & 0.001 & 0.503 & 0.613 \\
\hline $\mathrm{CaO}_{2}\left(\mathrm{~mL} \mathrm{O}_{2} \cdot \mathrm{dl}^{-1}\right)$ & $22.2 \pm 2.3$ & $19.3 \pm 2.0 \dagger$ & $21.7 \pm 4.2$ & $18.6 \pm 2.6 \dagger$ & 0.005 & 0.518 & 0.879 \\
\hline \multicolumn{8}{|l|}{ Cardiorespiratory } \\
\hline $\mathrm{BP}(\mathrm{mmHg})$ & $105.7 \pm 8.8$ & $102.3 \pm 8.2$ & $103.8 \pm 10.3$ & $99.3 \pm 8.8$ & 0.177 & 0.396 & 0.856 \\
\hline $\mathrm{HR}\left(\mathrm{b} \cdot \mathrm{min}^{-1}\right)$ & $74 \pm 11$ & $90 \pm 13+$ & $72 \pm 12$ & $95 \pm 19+$ & 0.001 & 0.729 & 0.229 \\
\hline $\mathrm{CO}\left(\mathrm{L} \cdot \mathrm{min}^{-1}\right)$ & $7.3 \pm 1.3$ & $7.1 \pm 1.3+$ & $8.1 \pm 1.9$ & $8.3 \pm 1.7 \dagger$ & 0.024 & 0.959 & 0.708 \\
\hline$\dot{\mathrm{V}} \mathrm{E}\left(\mathrm{L} \cdot \mathrm{min}^{-1}\right)$ & $17.3 \pm 2.9$ & $14.7 \pm 1.5 \dagger$ & $17.9 \pm 2.6$ & $16.1 \pm 2.2 \dagger$ & 0.005 & 0.276 & 0.550 \\
\hline$\dot{\mathrm{VO}} 2\left(\mathrm{ml} \cdot \mathrm{min}^{-1}\right)$ & $365 \pm 40$ & $376 \pm 56$ & $347 \pm 45$ & $383 \pm 57$ & 0.167 & 0.658 & 0.328 \\
\hline$\dot{\mathrm{V}} \mathrm{CO}_{2}\left(\mathrm{ml} \cdot \mathrm{min}^{-1}\right)$ & $340 \pm 46$ & $323 \pm 43$ & $304 \pm 41$ & $337 \pm 53$ & 0.579 & 0.481 & 0.099 \\
\hline RER & $0.92 \pm 0.05$ & $0.86 \pm 0.07 \dagger$ & $0.88 \pm 0.04$ & $0.88 \pm 0.05$ & 0.060 & 0.517 & 0.047 \\
\hline $\mathrm{SpO}_{2}(\%)$ & $98.8 \pm 1.1$ & $78.4 \pm 8.0 \dagger$ & $99.0 \pm 0.7$ & $83.9 \pm 4.3 \dagger$ & 0.001 & 0.054 & 0.074 \\
\hline \multicolumn{8}{|c|}{ Cerebral haemodynamics } \\
\hline $\operatorname{MCAv}\left(\mathrm{cm} \cdot \mathrm{s}^{-1}\right)$ & $63.1 \pm 16.9$ & $65.2 \pm 13.6$ & $54.4 \pm 9.6$ & $58.3 \pm 8.6$ & 0.302 & 0.134 & 0.808 \\
\hline Cerebral $\left[\mathrm{O}_{2} \mathrm{Hb}\right](\mu \mathrm{mol})$ & $0.9 \pm 3.0$ & $0.3 \pm 2.4$ & $1.4 \pm 2.6$ & $1.7 \pm 3.8$ & 0.321 & 0.888 & 0.649 \\
\hline Cerebral [HHb] ( $\mu \mathrm{mol})$ & $-0.2 \pm 1.5$ & $-0.5 \pm 2.2 \dagger$ & $2.4 \pm 3.9$ & $2.2 \pm 3.0+$ & 0.005 & 0.793 & 0.956 \\
\hline Cerebral [totHb] ( $\mu \mathrm{mol})$ & $0.7 \pm 3.7$ & $-0.1 \pm 4.1 \dagger$ & $3.8 \pm 4.7$ & $3.9 \pm 5.5+$ & 0.017 & 0.800 & 0.728 \\
\hline \multicolumn{8}{|l|}{ Muscle oxygenation } \\
\hline Muscle $\left[\mathrm{O}_{2} \mathrm{Hb}\right](\mu \mathrm{mol})$ & $1.2 \pm 2.3$ & $3.0 \pm 2.7$ & $3.4 \pm 4.5$ & $1.9 \pm 4.7$ & 0.651 & 0.906 & 0.174 \\
\hline Muscle $[\mathrm{HHb}](\mu \mathrm{mol})$ & $-0.6 \pm 3.6$ & $-0.5 \pm 2.5 \dagger$ & $1.9 \pm 3.3$ & $1.8 \pm 2.5+$ & 0.016 & 0.992 & 0.876 \\
\hline Muscle [totHb] ( $\mu \mathrm{mol})$ & $0.6 \pm 3.4$ & $2.5 \pm 3.2$ & $5.3 \pm 7.2$ & $3.7 \pm 5.1$ & 0.070 & 0.924 & 0.265 \\
\hline
\end{tabular}

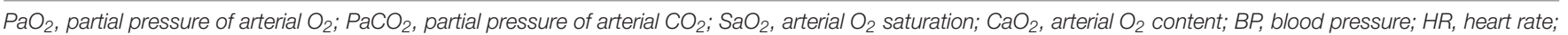

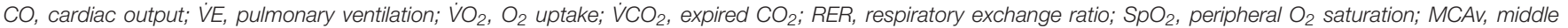
cerebral artery velocity; $\left[\mathrm{O}_{2} \mathrm{Hb}\right.$ ], oxy-hemoglobin concentration; [HHb], deoxy-hemoglobin concentration; [totHb], total hemoglobin concentration. Values are mean \pm SD. †different from normoxia ( $p<0.05)$. Bold text indicate significant main effect $(p<0.05)$. 
TABLE 3 | Arterial blood gases in Han Chinese and Tibetans during exercise at simulated sea-level and 5,000 m.

\begin{tabular}{|c|c|c|c|c|c|c|c|c|}
\hline$n$ & & \multicolumn{2}{|c|}{ Han Chinese } & \multicolumn{2}{|c|}{ Tibetan } & \multicolumn{3}{|c|}{ Main effects ( $p$-values) } \\
\hline \multirow{3}{*}{$\mathrm{PaO}_{2}(\mathrm{mmHg})$} & $70 W$ & $128.8 \pm 7.5$ & $45.4 \pm 2.9+$ & $126.9 \pm 10.1$ & $46.3 \pm 4.6+$ & 0.001 & 0.474 & 0.131 \\
\hline & $100 \mathrm{~W}$ & $121.9 \pm 9.3$ & $47.3 \pm 5.2 \dagger$ & $124.9 \pm 7.6$ & $46.9 \pm 3.8+$ & & & \\
\hline & Max & $124.3 \pm 8.4$ & $50.8 \pm 4.6+$ & $122.9 \pm 9.5$ & $49.5 \pm 3.5 \dagger$ & 0.001 & 0.658 & 0.962 \\
\hline \multirow[t]{4}{*}{$\mathrm{PaCO}_{2}(\mathrm{mmHg})$} & $70 \mathrm{~W}$ & $46.2 \pm 7.1$ & $33.2 \pm 4.6+$ & $42.7 \pm 4.7$ & $31.8 \pm 4.0 \dagger$ & 0.001 & 0.575 & 0.160 \\
\hline & $100 \mathrm{~W}$ & $45.5 \pm 7.7$ & $28.5 \pm 5.3+$ & $43.4 \pm 4.9$ & $29.5 \pm 4.4 \uparrow$ & & & \\
\hline & $130 \mathrm{~W}$ & $47.0 \pm 5.6$ & - & $42.2 \pm 5.9$ & $25.5 \pm 4.1 \dagger$ & & & \\
\hline & $160 \mathrm{~W}$ & - & - & $42.5 \pm 6.2$ & - & & & \\
\hline & $160 \mathrm{~W}$ & - & - & $7.22 \pm 0.04$ & - & & & \\
\hline & Max & $7.19 \pm 0.06$ & $7.29 \pm 0.03+$ & $7.19 \pm 0.05$ & $7.28 \pm 0.04 \uparrow$ & 0.001 & 0.988 & 0.954 \\
\hline \multirow[t]{5}{*}{$\mathrm{SaO}_{2}(\%)$} & $70 \mathrm{~W}$ & $98.2 \pm 0.4$ & $79.7 \pm 2.5 \dagger$ & $98.6 \pm 0.3$ & $81.9 \pm 4.5 \dagger$ & 0.001 & 0.286 & 0.121 \\
\hline & $100 \mathrm{~W}$ & $98.0 \pm 0.6$ & $80.0 \pm 4.6+$ & $98.4 \pm 0.2$ & $81.4 \pm 2.8+$ & & & \\
\hline & $130 \mathrm{~W}$ & $97.6 \pm 0.7$ & - & $98.2 \pm 0.3$ & $81.1 \pm 3.7 \dagger$ & & & \\
\hline & $160 W$ & - & - & $97.9 \pm 0.3$ & - & & & \\
\hline & Max & $97.6 \pm 0.8$ & $81.2 \pm 4.7 \dagger$ & $97.6 \pm 0.9$ & $80.5 \pm 3.2 \dagger$ & 0.001 & 0.804 & 0.752 \\
\hline \multirow[t]{4}{*}[\mathrm{Hb}]{$\left(\mathrm{g} \cdot \mathrm{L}^{-1}\right)$} & $70 \mathrm{~W}$ & $16.3 \pm 2.5$ & $17.6 \pm 1.0$ & $16.2 \pm 2.5$ & $16.6 \pm 1.2$ & 0.003 & 0.257 & 0.190 \\
\hline & $100 \mathrm{~W}$ & $17.0 \pm 2.1$ & $18.3 \pm 2.0$ & $16.1 \pm 2.3$ & $16.8 \pm 1.2$ & & & \\
\hline & $130 \mathrm{~W}$ & $17.1 \pm 1.8$ & - & $16.9 \pm 1.1$ & $17.7 \pm 0.8$ & & & \\
\hline & $160 \mathrm{~W}$ & - & - & $17.3 \pm 1.4$ & - & & & \\
\hline
\end{tabular}

$\mathrm{PaO}_{2}$, partial pressure of arterial $\mathrm{O}_{2} ; \mathrm{PaCO}_{2}$, partial pressure of arterial $\mathrm{CO}_{2} ; \mathrm{SaO}_{2}$, arterial $\mathrm{O}_{2}$ saturation; [ $\left.\mathrm{Hb}\right]$, hemoglobin concentration; CaO , arterial $\mathrm{O}_{2}$ content. Values are mean $\pm S D .{ }^{\dagger}$ different from normoxia $(p<0.05)$. Bold text indicate significant main effect $(p<0.05)$.

normoxia for all, Table 2). There was a trend for Tibetans to display higher resting $\mathrm{SpO}_{2}$ compared to the Han Chinese group $(F=3.9, p=0.054)$, particularly in hypoxia (interaction: $F=3.4, p=0.074$, Table 2). Post hoc pairwise analysis showed resting $\mathrm{SpO}_{2}$ was higher in Tibetans by $\sim 5.5 \%$ in hypoxia compared to Han $(d=0.8, p=0.011)$, but was not different in normoxia $(p=0.917)$. Hypoxia selectively lowered resting RER in Han Chinese (interaction: $F=4.6, p=0.047$ ). As a result, hypoxia lowered resting RER in Han Chinese by $\sim 0.054$ ( $d=0.8, p=0.009$ vs. normoxia), while no change was observed in Tibetans $(d<0.1, p=0.929)$.

For a given workload, hypoxia elevated HR by $\sim 10.2 \mathrm{~b} \cdot \mathrm{min}^{-1}$ $(F=5.8, d=0.5, p=0.017)$ and lowered $\mathrm{SpO}_{2}$ by $\sim 20.1 \%$ $(F=965.9, d=1.5, p<0.001), \dot{\mathrm{V}}_{2}$ by $\sim 283 \mathrm{ml}^{-m_{n}}{ }^{-1}(F=11.9$, $d=0.6, p=0.001)$ and $\dot{\mathrm{V} C O}{ }_{2}$ by $\sim 251 \mathrm{ml}^{-\mathrm{min}^{-1}}(F=80.1$, $d=0.5, p<0.001)$, but had no effects on CO or BP $(F \leq 0.5$, $p>0.05$, Figures 2, 3). We observed no significant group effects on $\mathrm{HR}, \mathrm{SpO}_{2}, \mathrm{~V}_{2}$ or $\mathrm{CO}$ during step-incremental cycling $(p>0.05)$. There was a group difference on RER during stepincremental cycling $(F=9.4, p=0.002)$, which was mediated by a non-significant group difference in $\dot{\mathrm{V}} \mathrm{CO}_{2}(F=6.0, d=0.2$, $p=0.054)$. In both normoxia and hypoxia, RER was lower in Tibetans by $\sim 0.097(d=1.1)$ throughout the step-incremental cycling compared to Han Chinese.

During recovery, hypoxia lowered $\mathrm{SpO}_{2}$ by $\sim 11.9 \%(F=161.2$, $d=1.8, p<0.001)$ compared to normoxia, but had no effects on HR $(F=0.0, p=0.872)$ or BP $(F=1.3, p=0.269$, Figure 2$)$. There were no group differences in cardiorespiratory parameters during recovery $(p>0.05)$.

During maximal effort, hypoxia lowered $\mathrm{SpO}_{2}$ by $\sim 15.6 \%$ $(F=62.5, d=1.5, p<0.001), \dot{\mathrm{V}} \mathrm{O}_{2}$ by $\sim 462 \mathrm{ml} \cdot \mathrm{min}^{-1}(F=18.2$, $d=0.9, p=0.001)), \dot{\mathrm{V} C O}{ }_{2}$ by $\sim 462 \mathrm{ml} \cdot \mathrm{min}^{-1}(F=15.9, d=0.8$, $p<0.001)$ and CO by $\sim 1.7 \mathrm{~L} \cdot \mathrm{min}^{-1}(F=5.5, d=0.6, p=0.031)$, but had no effect on HR or BP ( $p>0.05$, Figure 2,3$)$. Irrespective 


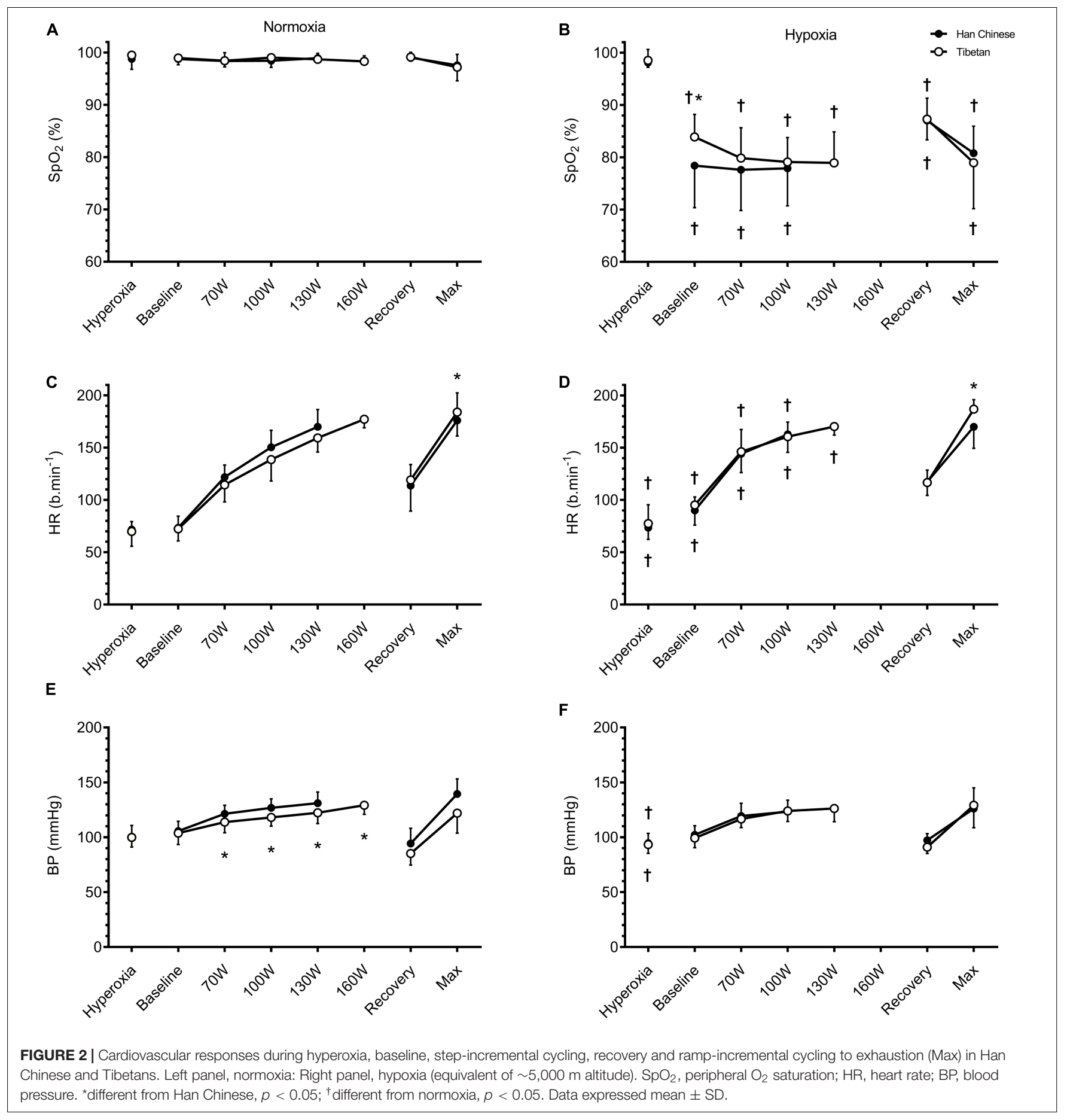

of the experimental conditions, Tibetans had higher HR (by $\sim 17.5$ b. min $^{-1}, F=6.3, p=0.017$, Figure 2) and CO at maximal exercise compared to Han Chinese (by $\sim 3.2 \mathrm{~L} \cdot \mathrm{min}^{-1}, F=13.6$, $d=1.1, p=0.002$ ).

\section{Energy Economy}

No condition or group effects were observed in EE, GE or EC during step-incremental cycling ( $p<0.05$, Table 4). Hypoxia lowered EE at maximal effort by $\sim 370 \mathrm{~J} . s^{-1}(F=10.5, d=1.9$, $p=0.005)$. Irrespective of the condition, GE was higher by $\sim 5 \%$, in Tibetans at maximal effort compared to Han Chinese $(F=10.7$, $d=1.1, p=0.004)$, while EC was higher by $\sim 0.18 \mathrm{~kJ} \cdot \mathrm{L}^{-1}(F=5.3$, $d=0.9, p=0.034$, Table 4).

\section{Muscle Tissue Oxygenation}

In both groups, hypobaric hypoxia elevated resting muscle [ $\mathrm{HHb}]$ by $\sim 2.4 \mu \mathrm{M}(F=6.4, d=0.8, p=0.016)$, but had no effects on muscle [totHb] $(F=3.4, p=0.070)$ or $\left[\mathrm{O}_{2} \mathrm{Hb}\right](F=0.2, p=0.651)$. 

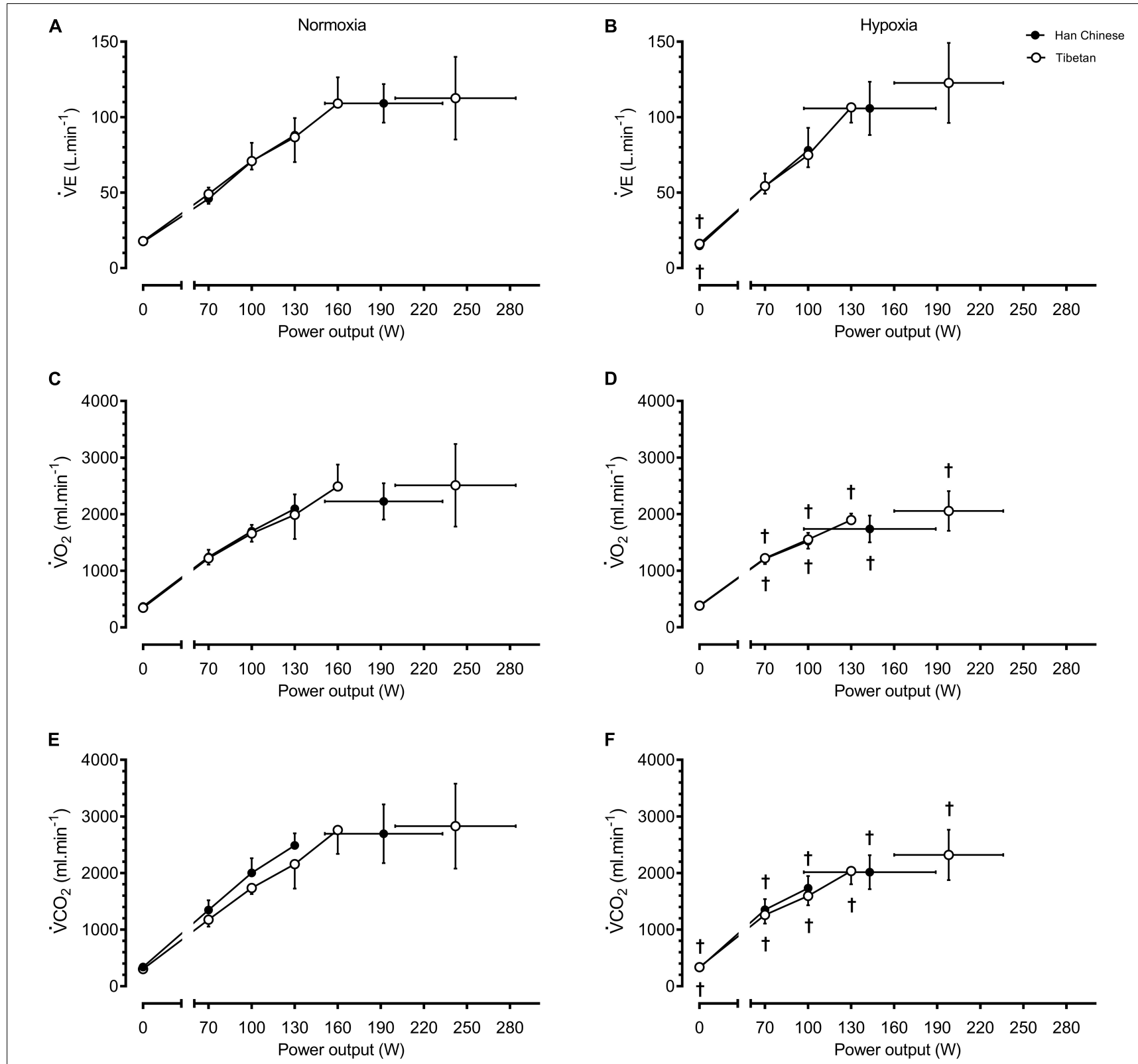

FIGURE 3 | Ventilation and metabolic responses during baseline, step-incremental cycling and maximal exertion (MAX) in Han Chinese and Tibetans. Left panel, normoxia: Right panel, hypoxia (equivalent of $\sim 5,000 \mathrm{~m}$ altitude). $\dot{\mathrm{V}}$, pulmonary ventilation; $\dot{\mathrm{VO}}_{2}, \mathrm{O}_{2}$ uptake; $\mathrm{V}_{\mathrm{CO}}$, expired $\mathrm{CO}_{2}$. ${ }^{*}$ different from Han $\mathrm{Chinese}$ $p<0.05$; ${ }^{\dagger}$ different from normoxia I, $p<0.05$. Data expressed mean $\pm \mathrm{SD}$.

No group effects were observed in resting muscle NIRS signals ( $p>0.05$, Table 2).

During step-incremental cycling, hypoxia lowered muscle $\left[\mathrm{O}_{2} \mathrm{Hb}\right]$ by $\sim 3.1 \mu \mathrm{M}$ in both groups $(F=10.7, d=0.5, p<0.001)$, and elevated muscle [HHb] by $\sim 4.7 \mu \mathrm{M}(F=15.1, d=0.5$, $p<0.001)$, while muscle [totHb] was unchanged $(F=2.6$, $p=0.108$, Figure 4). This hypoxia effect on exercising muscle $\left[\mathrm{O}_{2} \mathrm{Hb}\right]$ appeared to be limited to Tibetans (interaction: $F=23.3$, $p<0.001)$. Hypoxia lowered muscle $\left[\mathrm{O}_{2} \mathrm{Hb}\right]$ by $\sim 7.6 \mu \mathrm{M}$ in Tibetans $(d=1.0, p<0.001$ vs. normoxia) but not in Han Chinese $\left(d=0.3, p=0.298\right.$, Figure 4). As a result, muscle $\mathrm{O}_{2} \mathrm{Hb}$ was selectively lower in Tibetans during hypoxic exercise (by $\sim 6.7 \mu \mathrm{M}, d=0.9, p=0.003$ vs. Han Chinese), but not during normoxic exercise $(d=0.4, p=0.240$, Figure 4).

We observed an interaction between hypoxia and group on muscle [totHb] $(F=8.7, p=0.004)$. Post hoc analysis showed that hypoxia selectively increased muscle [totHb] in Han Chinese (by $\sim 4.6 \mu \mathrm{M}, d=0.6, p=0.003$ ), but not in Tibetans ( $d=0.2, p=0.325$, Figure 4). Nevertheless, this increase did not result in a significant difference in muscle [totHb] between the groups during exercise in hypoxia $(d=0.5$, $p=0.117)$. We found no significant group effects on muscle 
TABLE 4 | Energy economy in Han Chinese and Tibetans during exercise at simulated sea-level and 5,000 m.

\begin{tabular}{|c|c|c|c|c|c|c|c|c|}
\hline \multirow{3}{*}{$n$} & & \multicolumn{2}{|c|}{ Han Chinese } & \multicolumn{2}{|c|}{ Tibetan } & \multicolumn{3}{|c|}{ Main effects ( $p$-values) } \\
\hline & & \multicolumn{2}{|c|}{10} & \multicolumn{2}{|c|}{10} & \multirow[b]{2}{*}{ Condition } & \multirow[b]{2}{*}{ Group } & \multirow[b]{2}{*}{ Interaction } \\
\hline & & Normoxia & Hypoxia & Normoxia & Hypoxia & & & \\
\hline \multirow[t]{5}{*}{$\mathrm{EE}\left(\mathrm{J} \cdot \mathrm{s}^{-1}\right)$} & $70 W$ & $448 \pm 46$ & $440 \pm 40$ & $428 \pm 40$ & $435 \pm 24$ & 0.245 & 0.221 & 0.337 \\
\hline & $100 \mathrm{~W}$ & $626 \pm 43$ & $555 \pm 50$ & $592 \pm 46$ & $552 \pm 42$ & & & \\
\hline & $130 \mathrm{~W}$ & $773 \pm 82$ & - & $717 \pm 149$ & $681 \pm 48$ & & & \\
\hline & $160 \mathrm{~W}$ & - & - & $903 \pm 136$ & - & & & \\
\hline & Max & $829 \pm 133$ & $643 \pm 81^{\dagger}$ & $924 \pm 240$ & $751 \pm 129$ & 0.005 & 0.958 & 0.238 \\
\hline \multirow[t]{5}{*}{ GE (\%) } & $70 \mathrm{~W}$ & $14.9 \pm 1.7$ & $14.9 \pm 1.8$ & $16.5 \pm 1.6$ & $16.1 \pm 1.4$ & 0.129 & 0.397 & 0.494 \\
\hline & $100 \mathrm{~W}$ & $14.3 \pm 1.1$ & $16.6 \pm 2.1$ & $17.0 \pm 1.4$ & $18.2 \pm 1.4$ & & & \\
\hline & $130 \mathrm{~W}$ & $14.9 \pm 1.8$ & - & $19.1 \pm 5.2$ & $19.2 \pm 1.2$ & & & \\
\hline & $160 \mathrm{~W}$ & - & - & $18.2 \pm 3.7$ & - & & & \\
\hline & Max & $23.1 \pm 2.7$ & $24.1 \pm 4.2$ & $26.7 \pm 3.8^{\star}$ & $26.6 \pm 4.1^{\star}$ & 0.120 & 0.004 & 0.823 \\
\hline \multirow[t]{5}{*}{$\mathrm{EC}\left(\mathrm{kJ} . \mathrm{L}^{-1}\right)$} & $70 \mathrm{~W}$ & $0.81 \pm 0.09$ & $0.83 \pm 0.06$ & $0.83 \pm 0.08$ & $0.82 \pm 0.04$ & 0.875 & 0.398 & 0.671 \\
\hline & $100 \mathrm{~W}$ & $0.85 \pm 0.06$ & $0.95 \pm 0.09$ & $0.87 \pm 0.08$ & $0.93 \pm 0.07$ & & & \\
\hline & $130 \mathrm{~W}$ & $0.90 \pm 0.11$ & - & $0.99 \pm 0.28$ & $0.99 \pm 0.06$ & & & \\
\hline & $160 \mathrm{~W}$ & - & - & $0.96 \pm 0.21$ & - & & & \\
\hline & Max & $1.22 \pm 0.15$ & $1.26 \pm 0.22$ & $1.39 \pm 0.18^{*}$ & $1.43 \pm 0.23^{*}$ & 0.468 & 0.034 & 0.773 \\
\hline
\end{tabular}

$E E$, energy expenditure; GE, gross efficiency; $E C$, exercise economy. Values are mean $\pm S D .{ }^{\dagger}$ different from normoxia $(p<0.05)$. ${ }^{*}$ different from Han Chinese ( $\left.p<0.05\right)$. Bold text indicate significant main effect $(p<0.05)$.

[HHb] during step-incremental cycling $(F=0.1, p=0.778$, Figure 4). No significant condition or group effects were observed on muscle tissue NIRS signals during recovery or at maximal effort $(p>0.05)$.

\section{Cerebral Tissue Oxygenation and Hemodynamics}

When compared to normoxia, hypoxia lowered resting cerebral [HHb] in both groups by $\sim 2.6 \mu \mathrm{M}(F=10.3, d=0.9, p=0.005)$ and [totHb] by $\sim 3.5 \mu \mathrm{M}(F=6.9, d=0.7, p=0.017)$, but had no effect on resting MCAv $(F=1.1, p=0.302)$ or cerebral $\left[\mathrm{O}_{2} \mathrm{Hb}\right]$ $(F=1.0, p=0.321$, Table 2$)$. No group effects were observed in resting cerebral haemodynamics $(p>0.05$, Table 2$)$.

On average, hypoxia elevated cerebral [HHb] during stepincremental cycling by $\sim 3.7 \mu \mathrm{M}$ in both Tibetan and Han Chinese $(F=58.4, d=1.8)$, and lowered cerebral $\left[\mathrm{O}_{2} \mathrm{Hb}\right]$ by $\sim 7.9 \mu \mathrm{M}(F=67.8, d=1.6)$ and cerebral [totHb] by $\sim 4.1 \mu \mathrm{M}(F=12.4, d=0.6, p<0.001$ for all, Figure 5). Hypoxia also lowered MCAv by $\sim 8.4 \mathrm{~cm}^{-\mathrm{s}^{-1}}(F=29.6, d=0.5$, $p<0.001$ ), this reduction was greater in the Han Chinese group (interaction: $F=8.0, p=0.006$, Figure 5). In Han Chinese, hypoxia lowered MCAv by $\sim 12.8 \mathrm{~cm} . \mathrm{s}^{-1}$ during stepincremental cycling $(d=0.7, p<0.001)$, while hypoxia only tended to lower MCAv in Tibetans by $\sim 4.1 \mathrm{~cm} . \mathrm{s}^{-1}(d=0.4$, $p=0.055)$. Tibetans had lower MCAv compared to Han Chinese in normoxia (by $\sim 15.3 \mathrm{~cm} . \mathrm{s}^{-1}, d=0.8, p=0.039$ ), while they were not significantly different in hypoxia $(d=0.5, p=0.360$, Figure 5). We did not observe any significant group effects on cerebral $\left[\mathrm{O}_{2} \mathrm{Hb}\right](F=0.1, p=0.766),[\mathrm{HHb}](F=2.1$, $p=0.164)$ or $[$ totHb] during step-incremental cycling $(F=0.6$, $p=0.438$, Figure 5).

Hypoxia lowered cerebral $\left[\mathrm{O}_{2} \mathrm{Hb}\right]$ during recovery, by $\sim 3.9 \mu \mathrm{M}$ in both groups $(F=7.3, d=0.8, p=0.011$ vs. normoxia) and cerebral [totHb] by $\sim 4.3 \mu \mathrm{M}(F=8.6, d=0.8, p=0.006)$, compared to normoxia but not $\operatorname{MCAv}(F=2.4, p=0.140)$ or cerebral $[\mathrm{HHb}](F=1.2, p=0.283$, Figure 5$)$. We found no between-group differences in cerebral haemodynamics during recovery $(p>0.05$, Figure 5).

At maximal effort, hypoxia lowered MCAv by $\sim 13.8 \mathrm{~cm} . \mathrm{s}^{-1}$ $(F=17.6, d=1.0, p<0.001)$, cerebral $\left[\mathrm{O}_{2} \mathrm{Hb}\right]$ by $\sim 10.9 \mu \mathrm{M}$ $(F=54.5, d=1.3)$ and cerebral [totHb] by $\sim 6.9 \mu \mathrm{M}(F=13.0$, $d=0.8)$, and elevated cerebral [HHb] by $\sim 4.1 \mu \mathrm{M}(F=17.9$, $d=1.0, p<0.01$ for all vs. normoxia, Figure 5). We observed a group difference in cerebral $\left[\mathrm{O}_{2} \mathrm{Hb}\right](F=5.6$, $p=0.031$ ), but only in normoxia (interaction: $F=8.6$, $p=0.010)$. Compared to Han Chinese, cerebral $\left[\mathrm{O}_{2} \mathrm{Hb}\right]$ was higher in Tibetans at maximal effort in normoxia by $\sim 8.9 \mu \mathrm{M}$ $(d=1.1, p=0.001)$, but not in hypoxia $(d=0.0, p=0.962$, Figure 5). Similarly, there was a significant group effect on cerebral [totHb] $(F=5.4, p=0.033)$. As a result, hypoxia reduced cerebral [totHb] during maximal effort in Tibetans by $\sim 10.8 \mu \mathrm{M}(d=1.2, p=0.001$ vs. normoxia), but not in Han Chinese $(d=0.4, p=0.285$, Figure 5). No group effect was observed in cerebral $[\mathrm{HHb}]$ at maximal exercise $(F=1.5, p=0.235)$.

\section{DISCUSSION}

The underlying mechanisms responsible for Tibetans' superior work capacity at altitude remains unclear. We compared cerebral and muscle tissue oxygenation responses to step-incremental cycling and at maximal exercise during ramp incremental cycling between Tibetans and Han Chinese in normobaric normoxia and hypobaric hypoxia in a pressure-regulated 


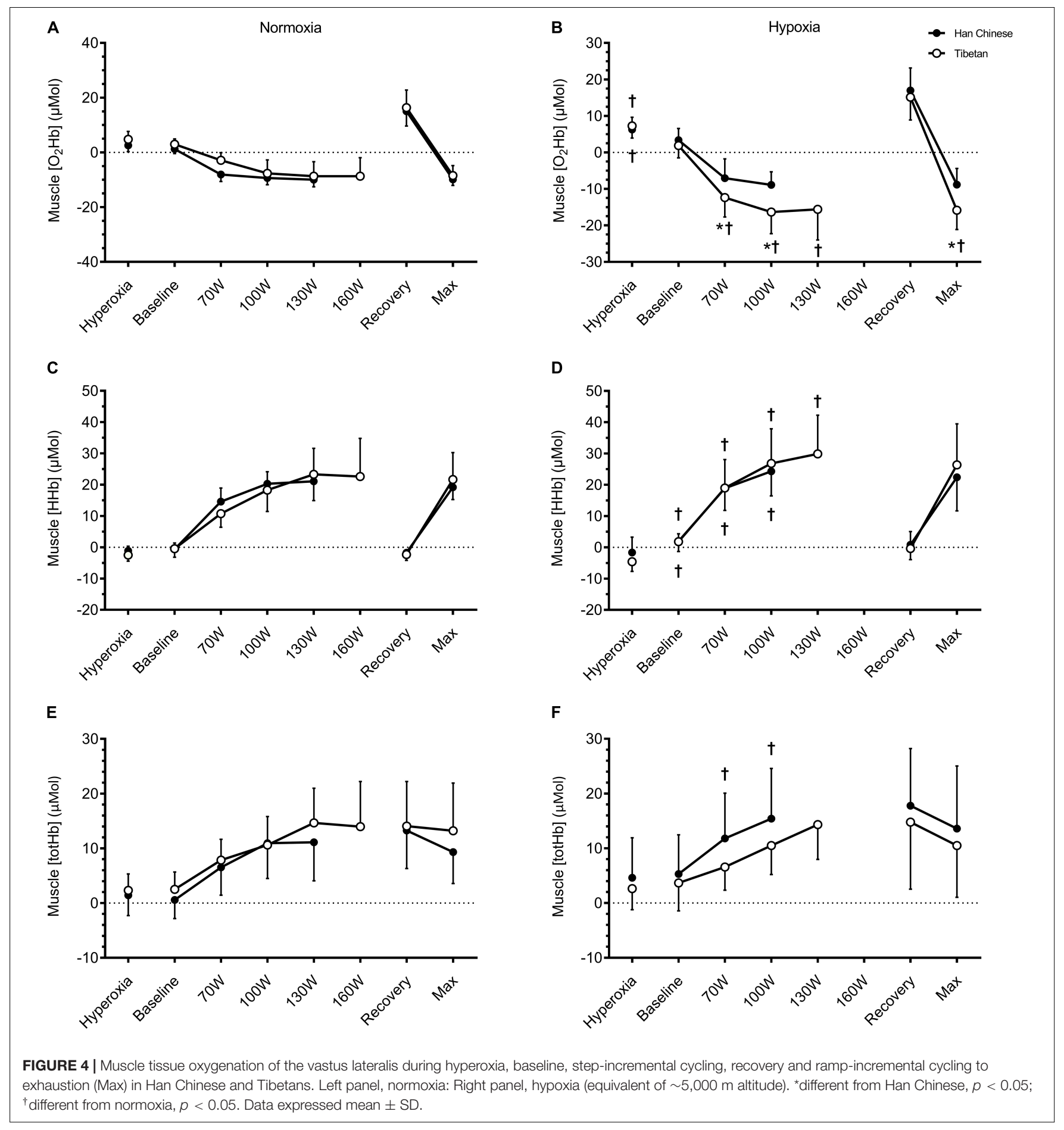

chamber, simulating altitudes equivalent to sea-level and $5,000 \mathrm{~m}$ altitude, respectively. Our main findings are that: (1) Irrespective of the condition, Tibetans consistently outperformed their Han Chinese counterparts and exhibited better economy at maximal exertion; (2) In normoxia, Tibetans displayed lower MCAv during submaximal exercise, yet achieved higher cerebral $\left[\mathrm{O}_{2} \mathrm{Hb}\right]$ and [tot $\left.\mathrm{Hb}\right]$ at maximal effort compared to Han Chinese; (3) In hypoxia, Tibetans displayed lower muscle $\left[\mathrm{O}_{2} \mathrm{Hb}\right]$ compared to the Han Chinese, which was not mediated by any differences in $\mathrm{SaO}_{2}$ or $\mathrm{CaO}_{2}$; and (4) For a given workload, Tibetans exhibited greater muscle desaturation during exercise in hypoxia, but not in normoxia. It follows that during exercise in hypoxia Tibetans as compared to Han Chinese seem to defend their brain oxygenation over muscle oxygenation without any obvious cost to performance. 


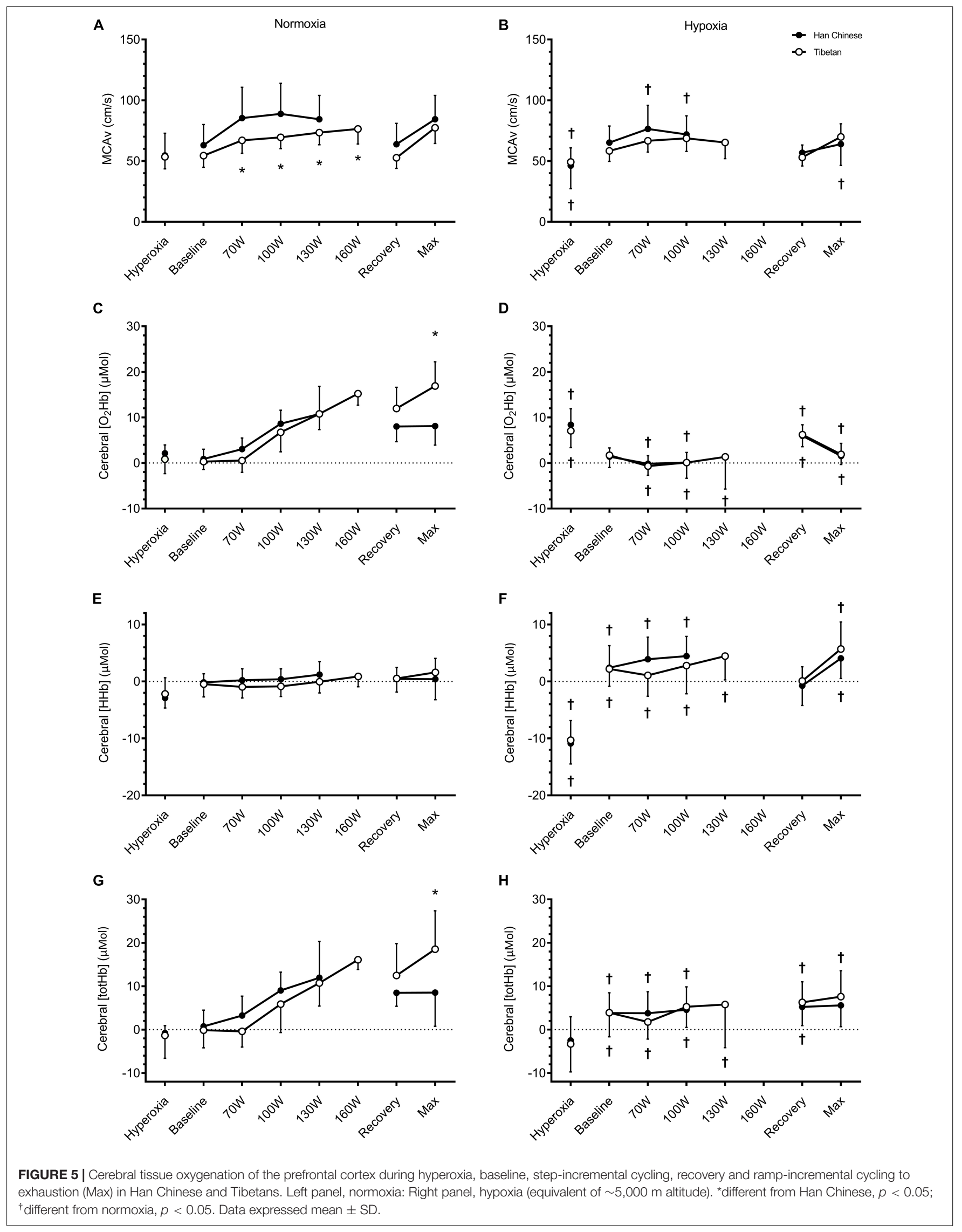


For a given workload, Subudhi et al. (2008) previously found comparable decreases in muscle $\left[\mathrm{O}_{2} \mathrm{Hb}\right]$ between exercise at low altitude and at $4,300 \mathrm{~m}$ in lowlanders, while muscle $[\mathrm{HHb}]$ was increased at high altitude, suggesting greater $\mathrm{O}_{2}$ extraction. Hypoxic training has been shown to enhance muscle $[\mathrm{HHb}]$ response to hypoxic exercise, while muscle $\left[\mathrm{O}_{2} \mathrm{Hb}\right]$ response was unaffected (Wang et al., 2010). Our Han Chinese confirmed these observations by showing unchanged muscle $\left[\mathrm{O}_{2} \mathrm{Hb}\right]$ responses to incremental exercise in hypobaric hypoxia when comparing with identical workloads in normoxia (Figure 4). Intriguingly, hypobaric hypoxia elicited a greater reduction in muscle $\left[\mathrm{O}_{2} \mathrm{Hb}\right]$ in Tibetans during exercise, while the increase in $[\mathrm{HHb}]$ was unaffected (Figure 4B). Since both $\mathrm{SaO}_{2}$ and $\mathrm{CaO}_{2}$ were similar in Tibetans and Han Chinese during hypoxic exercise (Table 3), this would suggest similar $\mathrm{O}_{2}$ extraction in Tibetans during hypoxic exercise (similar changes in $[\mathrm{HHb}]$ ), but lower muscle perfusion (less $\left[\mathrm{O}_{2} \mathrm{Hb}\right]$ and $[$ totHb]). Our muscle NIRS data allude to lower muscle $\mathrm{O}_{2}$ utilization in Tibetans for a given workload in hypoxia compared to Han Chinese. Furthermore, Tibetans achieved higher maximal workload despite similar $\dot{\mathrm{V}}_{2} \max$ in normoxia, and thus exhibited better exercise economy (Table 4). Our data support a previous study by $\mathrm{Ge}$ et al. (1994), who reported lower $\mathrm{V}_{2}$ consumption in Tibetans for a given submaximal exercise load compared to well-acclimatized ( $\sim 3.2$ years) Han Chinese at $4,700 \mathrm{~m}$, along with greater maximal work at a lower $\dot{\mathrm{V}} \mathrm{O}_{2}$ max. These findings allude to an enhanced muscle $\mathrm{O}_{2}$ economy, presumably due to a metabolic adaptation in Tibetans. Intriguingly, this enhanced muscle $\mathrm{O}_{2}$ economy appears to persist following descent to lower altitude (Marconi et al., 2005).

The underlying mechanism for this lower muscle perfusion and $\mathrm{O}_{2}$ utilization in Tibetans is unclear. One possible explanation is a higher reliance on glucose oxidation in Tibetans during hypoxic exercise. Glucose oxidation generates higher number of high-Englert phosphate bonds per mole of $\mathrm{O}_{2}$ (6.3 unit.mol $\mathrm{O}_{2}{ }^{-1}$ ) compared to fatty acid oxidation (4.1 unit.mol $\mathrm{O}_{2}{ }^{-1}$ ) (Kessler and Friedman, 1998). Therefore a higher reliance of glucose oxidation coupled with a lower reliance on fat oxidation increases the amount of ATP produced per molecule of $\mathrm{O}_{2}$ consumed, thereby enhances work-to- $\mathrm{O}_{2}$ ratio in the muscle (Hochachka et al., 1992; Hoppeler and Vogt, 2001). There is a predilection in Tibetans and Sherpas (a Nepalese ethnic group of Tibetan origin) toward carbohydrate oxidation and reduced reliance on intramyocellular lipids and lipid substrates (Hochachka et al., 1992; Kayser et al., 1996; Ge et al., 2015; Horscroft et al., 2017). The capacity of fatty acid oxidation is typically reduced at high altitude, mediated by decreased 3-hydroxyacyl-CoA dehydrogenase activity (Levett et al., 2012; Horscroft and Murray, 2014). Additional evidence for metabolic adaptation emerged from a genomic scan in Tibetan highlanders, where a haplotype of peroxisome proliferator-activated receptor $\alpha$ $(\operatorname{PPAR} \alpha)$ which was positively selected and associated with lower hematocrit (Simonson et al., 2010). PPAR $\alpha$ encodes the nuclear peroxisome proliferator activated receptor $\alpha$, which regulates fatty acid metabolism (Cullingford et al., 2002). At rest, we found acute hypoxic exposure selectively lowered RER in Han Chinese, presumably due to a shift toward fat utilization, while it was unchanged in Tibetans (Table 2). As reviewed by Gilbert-Kawai et al. (2014), our findings support a preferential use of carbohydratemetabolism in Tibetans.

A potential explanation for the ability to extract oxygen to a similar extent despite reduced perfusion and saturation is a reduced muscle diffusion path for $\mathrm{O}_{2}$ in Tibetans. In altitude-born Sherpas, Kayser et al. (1991) found muscle fiber cross-sectional area to be smaller, while capillary density was higher when compared to untrained lowlanders, but not different from that of fully acclimatized Caucasian climbers having spent two months at extreme altitude. This combination of smaller fiber cross-sectional area coupled with higher capillary density reduces the diffusion path for $\mathrm{O}_{2}$. Since these muscle characteristics were similar between Sherpas and acclimatized Caucasian climbers, it is likely the result of adaptation to extreme altitude per se, rather than a unique feature of the Himalayan natives. In addition, lower mitochondrial densities have been observed in both high altitude and lowland-dwelling Sherpas and Tibetans, resulting in a higher maximal $\mathrm{O}_{2}$ consumption-to-mitochondrial volume ratio (Kayser et al., 1991, 1996). Collectively, a shortened $\mathrm{O}_{2}$ diffusion path and higher mitochondrial $\mathrm{O}_{2}$ consumption would facilitate the higher muscle oxidative metabolism in Tibetans during hypoxic exercise.

Hyperventilation-induced hypocapnia during high-intensity exercise causes cerebral vasoconstriction, which can compromise cerebral $\mathrm{O}_{2}$ delivery and decrease cerebral tissue oxygenation (Fan and Kayser, 2016). In Han Chinese, we observed a steady rise in MCAv during step-incremental cycling in normoxia, which began to decline during workloads above $100 \mathrm{~W}$ (Figure 5A), likely the result from hypocapnia (Table 3 ). In contrast, Tibetans did not display the expected decline in MCAv at higher exercise intensity in normoxia, despite similar $\mathrm{PaCO}_{2}$ values (Table 3). Instead, MCAv, cerebral $\left[\mathrm{O}_{2} \mathrm{Hb}\right]$ and [tot $\left.\mathrm{Hb}\right]$ continued to rise in Tibetans during high intensity normoxic exercise (Figure 5). This difference in MCAv response resulted in higher cerebral $\left[\mathrm{O}_{2} \mathrm{Hb}\right]$ and [totHb] in Tibetans at maximal exercise compared to Han Chinese. This finding supports a blunted MCAv response to hypocapnia in Tibetans during heavy intensity exercise. Together with their higher cardiac output, this enabled them to maintain higher prefrontal tissue oxygenation at maximal effort. Since cerebral tissue deoxygenation has been proposed as one of the limiting factors of exercise performance (Nybo and Rasmussen, 2007; Amann and Kayser, 2009), the ability to maintain higher cerebral tissue oxygenation in normoxia could partly account for the superior aerobic performance in Tibetans.

The effect of hypoxia on the cerebral perfusion response to exercise varies between populations. In Tibetans living at 3,658 m, Huang et al. (1992) found a greater increase in internal carotid artery (ICA) blood velocity and estimated 
cerebral $\mathrm{O}_{2}$ delivery during incremental exercise compared to Han Chinese. They found ICA blood velocity returned toward resting values at maximal exercise in the Han Chinese group, while ICA blood velocity remained elevated in Tibetans. During incremental exercise at simulated $5,000 \mathrm{~m}$, we found no difference in MCAv and cerebral NIRS responses between Tibetan and Han Chinese (Figure 5). Compared to their normoxic values, hypoxia lowered MCAv during exercise by $\sim 23 \%$ in Han Chinese, while it only tended to lower it by $\sim 6 \%$ in Tibetans. As a result, the between-group difference in MCAv and cerebral NIRS responses to normoxic exercise were abolished during exercise in hypoxia. Together with the findings by Huang et al. (1992), our data indicates that Tibetans have a blunted cerebrovascular response to hypoxia compared to their Han Chinese counterparts, without adversely lowering cerebral tissue oxygenation.

We previously found higher $\mathrm{SpO}_{2}(\sim 9 \%)$ and $\mathrm{HR}(\sim 13$ $\mathrm{b} / \mathrm{min}$ ) in Tibetans during treadmill running in hypoxia compared to their Han Chinese counterparts (Kayser et al., 2019). Furthermore, iloprost inhalation improved hypoxic aerobic capacity in Han Chinese but not Tibetans, alluding to hypoxic pulmonary vasoconstriction and right ventricular function as limiting factors of hypoxic performance in Han. In the present study, we observed no between-group difference in $\mathrm{SpO}_{2}$ or maximal $\mathrm{HR}$ during hypoxic cycling exercise (Figure 2). Given that maximal $\mathrm{HR}$ is typically higher during incremental treadmill running compared to incremental cycling in trained and untrained individuals, and is influenced by training mode and postural position [see Millet et al. (2009) for review], we attribute the discrepant HR and $\mathrm{SpO}_{2}$ findings to the differences in exercise modality (cycling vs. treadmill running) and postural position (reclined and tilted vs. upright).

\section{Methodological Considerations}

There are several methodological considerations to be taken into account when interpreting our data. First, the $\mathrm{PaO}_{2}$ values we obtained in normoxic conditions were higher than expected $(\sim 130 \mathrm{mmHg}$, Table 2$)$. There are two possible explanations for this. Firstly, the simulated normoxic condition was mildly hyperbaric due to instrumental/chamber setting error. However, independent measure of chamber pressure indicates this was unlikely the case. Alternatively, there was a leak from the $\mathrm{O}_{2}$ masks used for the $100 \% \mathrm{O}_{2}$ breathing which resulted in $\mathrm{FIO}_{2}$ being $\sim 0.22-0.24$. This is the most likely explanation since we also observed slightly elevated $\mathrm{PaO}_{2}$ in the hypobaric hypoxic condition (Table 2). Accordingly, our findings should be interpreted as mild hyperoxia rather than normoxia. While the cause of the high $\mathrm{PaO}_{2}$ values is perplexing, we believe this does not affect our between-group comparisons since both Han Chinese and Tibetans exhibited high $\mathrm{PaO}_{2}$ (Table 2).

Second, muscle oximetry based on NIRS signals provides noninvasive and region-specific information of changes in $\left[\mathrm{O}_{2} \mathrm{Hb}\right]$ and $[\mathrm{HHb}]$ of the skeletal muscle tissue at rest and during exercise [see (Grassi and Quaresima, 2016; Perrey and Ferrari, 2017) for reviews], but there is much debate concerning the relative contributions of $\mathrm{Hb}$ and $\mathrm{Mb}$ to the muscle NIRS signals. At rest, $\mathrm{Mb}$ may contribute as much as $\sim 80 \%$ of the NIRS signal (Tran et al., 1999; Marcinek et al., 2007; Bendahan et al., 2017), but this value varies in relation to blood flow (as during exercise) and ambient $\mathrm{PO}_{2}$ (i.e., hypoxia) Spires et al. (2011). Given that Mb plays a crucial role in hypoxictolerance in deep-diving mammals (Fago and Jensen, 2015), and is expressed in greater proportions in Tibetans (Gelfi et al., 2004), it is possible the accentuated muscle tissue deoxygenation in Tibetans is the result of greater $\mathrm{Mb}$ deoxygenation during hypoxic exercise.

Finally, we recruited a small sample $(n=10$ in each group) of recreationally active individuals. Tibetans in this study consistently outperformed Han Chinese by $\sim 32 \%$ under both normoxic and hypoxic conditions, suggesting better efficiency during cycling in Tibetans. Wang et al. (2010) found hypoxic training does not affect muscle $\left[\mathrm{O}_{2} \mathrm{Hb}\right]$ response to hypoxic exercise nor cerebral NIRS parameters during normoxic exercise, despite a $\sim 50 \%$ improvement in maximal workload (+22 ml.min $\left.{ }^{-1} \cdot \mathrm{kg} \dot{\mathrm{VO}}_{2} \max \right)$ in their participants. Recently, Caen et al. (2019) showed aerobic training increased the amplitude of muscle tot $\mathrm{Hb}$ and $\mathrm{HHb}$ responses during incremental exercise in normoxia, indicating improved $\mathrm{O}_{2}$ availability and muscle $\mathrm{O}_{2}$ extraction with improved fitness. Meanwhile, we found comparable muscle tissue oxygenation responses between Han Chinese and Tibetans during incremental exercise in normoxia, and lower muscle $\mathrm{O}_{2} \mathrm{Hb}$ and tot $\mathrm{Hb}$ in Tibetans during hypoxic exercise. We contend that the between-group differences in muscle tissue oxygenation during incremental exercise is due to ethnicity rather than fitness.

\section{CONCLUSION}

We found distinct differences between Tibetans and Han Chinese in muscle and brain tissue oxygenation changes during exercise. We found Tibetans exhibited a blunted cerebrovascular response to hypocapnia during normoxic exercise. This combined with a higher heart rate (and cardiac output) enabled them to maintain a higher cerebral tissue oxygenation at maximal effort compared to Han Chinese. During hypoxic exercise, we found evidence of greater muscle tissue deoxygenation in Tibetans for a given workload, which we interpret as enhanced muscle $\mathrm{O}_{2}$ extraction. Tibetans consistently outperformed their Han Chinese counterpart in both normoxic and hypoxic conditions, exhibiting better energy economy at exercise exertion. For the first time, our data demonstrate that Tibetans can maintain higher cerebral tissue oxygenation during maximal normoxic exercise and enhance muscle $\mathrm{O}_{2}$ utilization during hypoxic exercise. Whether these muscular and cerebrovascular responses account for their superior aerobic performance warrants further investigation. 


\section{DATA AVAILABILITY STATEMENT}

The raw data supporting the conclusions of this article will be made available by the authors, without undue reservation.

\section{ETHICS STATEMENT}

The studies involving human participants were reviewed and approved by the University of Oregon Institutional Review Board and the Qinghai High Altitude Medical Science Institutional Committee. The patients/participants provided their written informed consent to participate in this study.

\section{AUTHOR CONTRIBUTIONS}

J-LF, TW, AL, and BK contributed to the conception and design of the study. J-LF, TW, LN, WLB, AL, and BK performed the data collection. J-LF, AL, and BK carried out the analysis, carried

\section{REFERENCES}

Allemann, Y., Hutter, D., Lipp, E., Sartori, C., Duplain, H., Egli, M., et al. (2006). Patent foramen ovale and high-altitude pulmonary edema. JAMA 296, 29542958. doi: 10.1001/jama.296.24.2954

Amann, M., Eldridge, M. W., Lovering, A. T., Stickland, M. K., Pegelow, D. F., and Dempsey, J. A. (2006). Arterial oxygenation influences central motor output and exercise performance via effects on peripheral locomotor muscle fatigue in humans. J. Physiol. 575 (Pt 3), 937-952.

Amann, M., and Kayser, B. (2009). Nervous system function during exercise in hypoxia. High Alt. Med. Biol. 10, 149-164. doi: 10.1089/ham.2008.1105

Amann, M., Romer, L. M., Subudhi, A. W., Pegelow, D. F., and Dempsey, J. A. (2007). Severity of arterial hypoxaemia affects the relative contributions of peripheral muscle fatigue to exercise performance in healthy humans. J. Physiol. 581 (Pt 1), 389-403.

Bendahan, D., Chatel, B., and Jue, T. (2017). Comparative NMR and NIRS analysis of oxygen-dependent metabolism in exercising finger flexor muscles. Am. J. Physiol. Regul. Integr. Comp. Physiol. 313, R740-R753. doi: 10.1152/ajpregu. 00203.2017

Brenner, R., Pratali, L., Rimoldi, S. F., Murillo Jauregui, C. X., Soria, R., Rexhaj, E., et al. (2015). Exaggerated pulmonary hypertension and right ventricular dysfunction in high-altitude dwellers with patent foramen ovale. Chest 147, 1072-1079. doi: 10.1378/chest.14-1353

Caen, K., Vermeire, K., Pogliaghi, S., Moerman, A., Niemeijer, V., Bourgois, J. G., et al. (2019). Aerobic interval training impacts muscle and brain oxygenation responses to incremental exercise. Front. Physiol. 10:1195. doi: 10.3389/fphys. 2019.01195

Cohen, J. (1977). Statistical Power Analysis for the Behavioral Sciences. New York, NY: Academic Press.

Cullingford, T. E., Dolphin, C. T., and Sato, H. (2002). The peroxisome proliferator-activated receptor alpha-selective activator ciprofibrate upregulates expression of genes encoding fatty acid oxidation and ketogenesis enzymes in rat brain. Neuropharmacology 42, 724-730. doi: 10.1016/s0028-3908(02)0 0014- $\mathrm{x}$

Duncan, A., Meek, J. H., Clemence, M., Elwell, C. E., Tyszczuk, L., Cope, M., et al. (1995). Optical pathlength measurements on adult head, calf and forearm and the head of the newborn infant using phase resolved optical spectroscopy. Phys. Med. Biol. 40, 295-304. doi: 10.1088/0031-9155/40/2/007

Elliott, J. E., Laurie, S. S., Kern, J. P., Beasley, K. M., Goodman, R. D., Kayser, B., et al. (2015). AltitudeOmics: impaired pulmonary gas exchange efficiency and blunted ventilatory acclimatization in humans with patent foramen ovale after 16 days at 5,260 m. J. Appl. Physiol. (1985) 118, 1100-1112. doi: 10.1152/ japplphysiol.00879.2014 out the interpretation of the data, and contributed to the revision of the manuscript. J-LF drafted the manuscript and prepared the figures. All authors approved the final version of the manuscript.

\section{FUNDING}

We acknowledge the support of the Sino Swiss Science and Technology Cooperation Program (EG 06-092009) and a Chinese research grant (973-2012CB18202).

\section{ACKNOWLEDGMENTS}

We thank the participants for their participation, the technical support personnel of the hypobaric chamber at the National Key Laboratory of High Altitude Medicine in Xining for their expert help and the doctors and nurses at the Qinghai People's Hospital in Xining for their assistances.

Erzurum, S. C., Ghosh, S., Janocha, A. J., Xu, W., Bauer, S., Bryan, N. S., et al. (2007). Higher blood flow and circulating NO products offset high-altitude hypoxia among Tibetans. Proc. Natl. Acad. Sci. U.S.A. 104, 17593-17598. doi: $10.1073 /$ pnas.0707462104

Fago, A., and Jensen, F. B. (2015). Hypoxia tolerance, nitric oxide, and nitrite: lessons from extreme animals. Physiology (Bethesda) 30, 116-126. doi: 10.1152/ physiol.00051.2014

Fan, J. L., and Kayser, B. (2016). Fatigue and exhaustion in hypoxia: the role of cerebral oxygenation. High Alt. Med. Biol. 17, 72-84. doi: 10.1089/ham.2016. 0034

Foster, G. E., Ainslie, P. N., Stembridge, M., Day, T. A., Bakker, A., Lucas, S. J., et al. (2014). Resting pulmonary haemodynamics and shunting: a comparison of sea-level inhabitants to high altitude Sherpas. J. Physiol. 592(Pt 6), 1397-1409. doi: 10.1113/jphysiol.2013.266593

Ge, R. L., Chen, Q. H., Wang, L. H., Gen, D., Yang, P., Kubo, K., et al. (1994). Higher exercise performance and lower VO2max in tibetan than han residents at 4,700 m altitude. J. Appl. Physiol. 77, 684-691.

Ge, R. L., Simonson, T. S., Gordeuk, V., Prchal, J. T., and McClain, D. A. (2015). Metabolic aspects of high-altitude adaptation in Tibetans. Exp. Physiol. 100, 1247-1255. doi: 10.1113/EP085292

Gelfi, C., De Palma, S., Ripamonti, M., Eberini, I., Wait, R., Bajracharya, A., et al. (2004). New aspects of altitude adaptation in Tibetans: a proteomic approach. FASEB J. 18, 612-614. doi: 10.1096/fj.03-10 77 fje

Gilbert-Kawai, E. T., Milledge, J. S., Grocott, M. P., and Martin, D. S. (2014). King of the mountains: tibetan and Sherpa physiological adaptations for life at high altitude. Physiology (Bethesda) 29, 388-402. doi: 10.1152/physiol.00018. 2014

Grassi, B., and Quaresima, V. (2016). Near-infrared spectroscopy and skeletal muscle oxidative function in vivo in health and disease: a review from an exercise physiology perspective. J. Biomed. Opt. 21, 091313. doi: 10.1117/1.JBO. 21.9.091313

Hochachka, P. W., Stanley, C., McKenzie, D. C., Villena, A., and Monge, C. (1992). Enzyme mechanisms for pyruvate-to-lactate flux attenuation: a study of Sherpas, Quechuas, and hummingbirds. Int. J. Sports Med. 13(Suppl. 1), S119-S122. doi: 10.1055/s-2007-102 4613

Hoppeler, H., and Vogt, M. (2001). Muscle tissue adaptations to hypoxia. J. Exp. Biol. 204(Pt 18), 3133-3139.

Horscroft, J. A., Kotwica, A. O., Laner, V., West, J. A., Hennis, P. J., Levett, D. Z. H., et al. (2017). Metabolic basis to Sherpa altitude adaptation. Proc. Natl. Acad. Sci. U.S.A. 114, 6382-6387. doi: 10.1073/pnas.17005 27114 
Horscroft, J. A., and Murray, A. J. (2014). Skeletal muscle energy metabolism in environmental hypoxia: climbing towards consensus. Extrem. Physiol. Med. 3:19. doi: 10.1186/2046-7648-3-19

Huang, S. Y., Sun, S., Droma, T., Zhuang, J., Tao, J. X., McCullough, R. G., et al. (1992). Internal carotid arterial flow velocity during exercise in Tibetan and Han residents of Lhasa (3,658 m). J. Appl. Physiol. 73, 2638-2642.

Kayser, B., Fan, J. L., Nan, L., Bang, W. L., Bianba, and Wu, T. (2019). Iloprost improves running performance at 5,000m in Han but not in Tibetans. Curr. Issues Sport Sci. 4:002. doi: 10.15203/CISS_2019.002

Kayser, B., Hoppeler, H., Claassen, H., and Cerretelli, P. (1991). Muscle structure and performance capacity of Himalayan Sherpas. J. Appl. Physiol. (1985) 70, 1938-1942.

Kayser, B., Hoppeler, H., Desplanches, D., Marconi, C., Broers, B., and Cerretelli, P. (1996). Muscle ultrastructure and biochemistry of lowland Tibetans. J. Appl. Physiol. (1985) 81, 419-425.

Kelman, G. R., and Nunn, J. F. (1966). Nomograms for correction of blood PO2, PCO2, pH, and base excess for time and temperature. J. Appl. Physiol. 21, 1484-1490.

Kessler, G., and Friedman, J. (1998). Metabolism of fatty acids and glucose. Circulation 98:1351.

Kjaer, M., Hanel, B., Worm, L., Perko, G., Lewis, S. F., Sahlin, K., et al. (1999). Cardiovascular and neuroendocrine responses to exercise in hypoxia during impaired neural feedback from muscle. Am. J. Physiol. 277(1 Pt 2), R76-R85.

Levett, D. Z., Radford, E. J., Menassa, D. A., Graber, E. F., Morash, A. J., Hoppeler, H., et al. (2012). Acclimatization of skeletal muscle mitochondria to highaltitude hypoxia during an ascent of Everest. FASEB J. 26, 1431-1441. doi: 10.1096/fj.11- 197772

Lovering, A. T., Stickland, M. K., Amann, M., O’Brien, M. J., Hokanson, J. S., and Eldridge, M. W. (2011). Effect of a patent foramen ovale on pulmonary gas exchange efficiency at rest and during exercise. J. Appl. Physiol. (1985) 110, 1354-1361. doi: 10.1152/japplphysiol.01246.2010

Marcinek, D. J., Amara, C. E., Matz, K., Conley, K. E., and Schenkman, K. A. (2007). Wavelength shift analysis: a simple method to determine the contribution of hemoglobin and myoglobin to in vivo optical spectra. Appl. Spectrosc. 61, 665-669. doi: 10.1366/000370207781269819

Marconi, C., Marzorati, M., and Cerretelli, P. (2006). Work capacity of permanent residents of high altitude. High Alt. Med. Biol. 7, 105-115. doi: 10.1089/ham. 2006.7.105

Marconi, C., Marzorati, M., Sciuto, D., Ferri, A., and Cerretelli, P. (2005). Economy of locomotion in high-altitude Tibetan migrants exposed to normoxia. J. Physiol. 569(Pt 2), 667-675. doi: 10.1113/jphysiol.2005.094979

Millet, G. P., Vleck, V. E., and Bentley, D. J. (2009). Physiological differences between cycling and running: lessons from triathletes. Sports Med. 39, 179-206. doi: 10.2165/00007256-200939030-00002

Millet, G. Y., Muthalib, M., Jubeau, M., Laursen, P. B., and Nosaka, K. (2012). Severe hypoxia affects exercise performance independently of afferent feedback and peripheral fatigue. J. Appl. Physiol. (1985) 112, 1335-1344. doi: 10.1152/ japplphysiol.00804.2011

Moseley, L., and Jeukendrup, A. E. (2001). The reliability of cycling efficiency. Med. Sci. Sports Exerc. 33, 621-627. doi: 10.1097/00005768-200104000-00017

Nybo, L., and Rasmussen, P. (2007). Inadequate cerebral oxygen delivery and central fatigue during strenuous exercise. Exerc. Sport Sci. Rev. 35, 110-118.

Peltonen, J. E., Paterson, D. H., Shoemaker, J. K., Delorey, D. S., Dumanoir, G. R., Petrella, R. J., et al. (2009). Cerebral and muscle deoxygenation, hypoxic ventilatory chemosensitivity and cerebrovascular responsiveness during incremental exercise. Respir. Physiol. Neurobiol. 169, 24-35.
Perrey, S., and Ferrari, M. (2017). Muscle oximetry in sports science: a systematic review. Sports Med. 48, 597-616. doi: 10.1007/s40279-017-0820-1

Rasmussen, P., Dawson, E. A., Nybo, L., van Lieshout, J. J., Secher, N. H., and Gjedde, A. (2007). Capillary-oxygenation-level-dependent near-infrared spectrometry in frontal lobe of humans. J. Cereb. Blood Flow Metab. 27, 1082-1093. doi: 10.1038/sj.jcbfm.9600416

Rasmussen, P., Nielsen, J., Overgaard, M., Krogh-Madsen, R., Gjedde, A., Secher, N. H., et al. (2010). Reduced muscle activation during exercise related to brain oxygenation and metabolism in humans. J. Physiol. 588(Pt 11), 1985-1995.

Rupp, T., and Perrey, S. (2009). Effect of severe hypoxia on prefrontal cortex and muscle oxygenation responses at rest and during exhaustive exercise. Adv. Exp. Med. Biol. 645, 329-334. doi: 10.1007/978-0-387-85998-9_49

Severinghaus, J. W. (1966). Blood gas calculator. J. Appl. Physiol. 21, 1108-1116.

Simonson, T. S., Yang, Y., Huff, C. D., Yun, H., Qin, G., Witherspoon, D. J., et al. (2010). Genetic evidence for high-altitude adaptation in Tibet. Science 329, 72-75. doi: 10.1126/science.1189406

Smith, K. J., and Billaut, F. (2010). Influence of cerebral and muscle oxygenation on repeated-sprint ability. Eur. J. Appl. Physiol. 109, 989-999. doi: 10.1007/s00421010-1444- 4

Spires, J., Lai, N., Zhou, H., and Saidel, G. M. (2011). Hemoglobin and myoglobin contributions to skeletal muscle oxygenation in response to exercise. Adv. Exp. Med. Biol. 701, 347-352. doi: 10.1007/978-1-4419-7756-4_47

Subudhi, A. W., Dimmen, A. C., and Roach, R. C. (2007). Effects of acute hypoxia on cerebral and muscle oxygenation during incremental exercise. J. Appl. Physiol. 103, 177-183.

Subudhi, A. W., Lorenz, M. C., Fulco, C. S., and Roach, R. C. (2008). Cerebrovascular responses to incremental exercise during hypobaric hypoxia: effect of oxygenation on maximal performance. Am. J. Physiol. Heart Circ. Physiol. 294, H164-H171. doi: 10.1152/ajpheart.01104.2007

Sullivan, G. M., and Feinn, R. (2012). Using Effect Size-or Why the P Value Is Not Enough. J Grad Med Educ 4, 279-282. doi: 10.4300/JGME-D-12-00156.1

Tran, T. K., Sailasuta, N., Kreutzer, U., Hurd, R., Chung, Y., Mole, P., et al. (1999). Comparative analysis of NMR and NIRS measurements of intracellular PO2 in human skeletal muscle. Am. J. Physiol. 276(6 Pt 2), R1682-R1690.

Vogiatzis, I., Louvaris, Z., Habazettl, H., Athanasopoulos, D., Andrianopoulos, V., Cherouveim, E., et al. (2011). Frontal cerebral cortex blood flow, oxygen delivery and oxygenation during normoxic and hypoxic exercise in athletes. J. Physiol. 589(Pt 16), 4027-4039. doi: 10.1113/jphysiol.2011.210880

Wang, J. S., Wu, M. H., Mao, T. Y., Fu, T. C., and Hsu, C. C. (2010). Effects of normoxic and hypoxic exercise regimens on cardiac, muscular, and cerebral hemodynamics suppressed by severe hypoxia in humans. J. Appl. Physiol. (1985) 109, 219-229. doi: 10.1152/japplphysiol.00138.2010

Wu, T., and Kayser, B. (2006). High altitude adaptation in Tibetans. High Alt. Med. Biol. 7, 193-208. doi: 10.1089/ham.2006.7.193

Wu, T., Li, S., and Ward, M. P. (2005). Tibetans at extreme altitude. Wilderness Environ. Med. 16, 47-54.

Conflict of Interest: The authors declare that the research was conducted in the absence of any commercial or financial relationships that could be construed as a potential conflict of interest.

Copyright (c) 2021 Fan, Wu, Lovering, Nan, Bang and Kayser. This is an open-access article distributed under the terms of the Creative Commons Attribution License (CC BY). The use, distribution or reproduction in other forums is permitted, provided the original author(s) and the copyright owner(s) are credited and that the original publication in this journal is cited, in accordance with accepted academic practice. No use, distribution or reproduction is permitted which does not comply with these terms. 